\title{
ON ZETA FUNCTIONS ASSOCIATED TO SYMMETRIC MATRICES, II: FUNCTIONAL EQUATIONS AND SPECIAL VALUES
}

\author{
TOMOYOSHI IBUKIYAMA AND HIROSHI SAITO
}

\begin{abstract}
New simple functional equations of zeta functions of the prehomogeneous vector spaces consisting of symmetric matrices are obtained, using explicit forms of zeta functions in the previous paper, Part I, and real analytic Eisenstein series of half-integral weight. When the matrix size is 2, our functional equations are identical with the ones by Shintani, but we give here an alternative proof. The special values of the zeta functions at nonpositive integers and the residues are also explicitly obtained. These special values, written by products of Bernoulli numbers, are used to give the contribution of "central" unipotent elements in the dimension formula of Siegel cusp forms of any degree. These results lead us to a conjecture on explicit values of dimensions of Siegel cusp forms of any torsion-free principal congruence subgroups of the symplectic groups of general degree.
\end{abstract}

\section{Contents}

0. Introduction . . . . . . . . . . . . . . . . . . . . 265

1. Results on functional equations, poles, and residues . . . . . . . . . . . 268

2. Fourier coefficients of Eisenstein series . . . . . . . . . . . . . . . . 274

3. Dirichlet series associated to Eisenstein series . . . . . . . . . . . . . . . 280

4. Proof of functional equations for $n \geq 3 \ldots \ldots \ldots . \ldots \ldots$

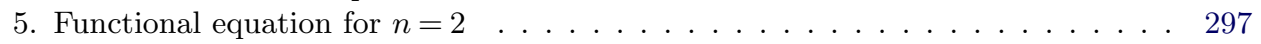

6. Special values of zeta functions and dimensions of Siegel cusp forms . . . . . . . 310

Acknowledgments . . . . . . . . . . . . . . . . . . . . 315

References . . . . . . . . . . . . . . . . . . . . . . . . . 315

\section{$\S 0$. Introduction}

In a previous paper [7], we gave an explicit formula for zeta functions of the prehomogeneous vector spaces consisting of symmetric matrices. In

Received July 30, 2011. Revised February 27, 2012. Accepted May 2, 2012.

2010 Mathematics Subject Classification. Primary 11F46, 11S90; Secondary 11E12, $11 \mathrm{R} 99$.

Ibukiyama's work was partially supported by Japan Society for the Promotion of Science Grant-in-Aid for Scientific Research no. 21244001.

(C) 2012 by The Editorial Board of the Nagoya Mathematical Journal 
this article, we give functional equations of these zeta functions. We also give special values of the zeta functions at nonpositive integers, poles, and residues. As an application, we give the contribution of "central unipotent" elements to the dimension of the space $S_{k}\left(\Gamma_{n}(N)\right)$ of Siegel cusp forms of weight $k$ of degree $n$ belonging to the principal congruence subgroup $\Gamma_{n}(N)$. This leads us to an explicit conjecture on dimensions of $S_{k}\left(\Gamma_{n}(N)\right)$ for $k>2 n$ and $N \geq 3$ for arbitrary $n$ as announced in [6].

Historically, functional equations for zeta functions associated with a large class of prehomogeneous vector spaces were investigated by Sato and Shintani [17], and the special cases treated in this paper were studied separately by Shintani [21], since it contains subtle points beyond the general theory. The functional equations treated there are between vectors of zeta functions. Indeed, in the above theory in [21], Shintani's functional equation for the prehomogeneous vector space of $n \times n$ symmetric matrices, the space which we treat here, is between $(n+1)$-dimensional vectors each of whose components is the zeta function associated with each real orbit of the prehomogeneous vector space. Later, Satake and Faraut [14], [13] simplified these functional equations by giving a kind of diagonalization. Since we have given the zeta functions explicitly in the previous paper [7], we can calculate everything explicitly, and we can show that, among $n+1$ components, there exist only two or three different zeta functions, if $n$ is odd or even, respectively, and that the functional equation is essentially between 2or 3-dimensional vectors of zeta functions. Hence, our functional equations are much simpler than the previously known ones in the general theory. Actually, we give here a more direct proof of the functional equations independently of any arguments by Shintani [21] or Satake and Faraut [14]. The diagonalization of the functional equations is essentially given by Riemann zeta functions when $n$ is odd. In fact, when $n$ is odd, the zeta functions are described by the Riemann zeta functions, so the functional equations are easily obtained. When $n$ is even, our argument is based on the functional equations of the Mellin transform of real analytic Eisenstein series of half-integral weights, and we obtain the results for $n \geq 4$ by some standard arguments. In this case, we do not write the diagonalization explicitly here since it contains nonelementary special functions and since nondiagonal functional equations are much simpler, but it is obviously contained in our arguments. The case $n=2$ is the pathological case, since the volumes $\mu(x)$ of the stabilizer of symmetric matrices $x$ used in the definition of our zeta functions for $n \geq 3$ are not finite for some $x$ when $n=2$. Hence, we need a 
modification of the definition of the zeta functions to obtain a good functional equation. Such modification has been done first by Shintani [21] and later by Sato [16] in a more intrinsic way. Here we give the third alternative proof of this functional equation for $n=2$ from our standpoint. The prototype of the proof of this style is found in Sturm [23] for the easier half of our cases. For all $n \geq 1$, this direct approach allows us to evaluate special values, locations of poles, and the residues of our zeta functions. This leads us to an application to dimension formulas of Siegel cusp forms of general degree.

This paper is organized as follows. In Section 1, we state functional equations, poles, and residues of our zeta functions for $n \geq 3$. In Section 2, we review the Fourier coefficients of the real analytic Eisenstein series of halfintegral weight, which is more or less known by [20], [3], [27], [23], and [4]. In Section 3, we introduce the Cohen-type real analytic Eisenstein series for a general parameter. For even $n \geq 4$, we use it to show the functional equation between Dirichlet series $D_{n}^{*}(s, \delta)$ and $D_{n}(s, \delta)$ which appear in some parts of our zeta functions. We also give an alternative proof of the functional equation between certain Dirichlet series of two variables associated to binary quadratic forms which was first proved by Shintani [21]. In Section 4 , we prove the functional equations for $n \geq 3$. In Section 5 , we assume that $n=2$ and give the definition of zeta functions and an alternative proof of functional equations described above. In Sections 3 and 5, we need some calculation on the Mellin transform of real analytic Eisenstein series with parameters. Goldfeld and Hoffstein [4] calculated this kind of Mellin transform for completely different purposes from ours. Our calculation is very similar to theirs in several points, but the details and aims are different. So, we write the proofs in detail here. The results in Section 6 were announced in [6], and we give here the proofs. We evaluate the values of our zeta functions at nonpositive integers. As an application, we give the contribution of central unipotent conjugacy classes to the dimension of Siegel cusp forms. Based on these results, we propose a conjecture for explicit dimension formulas for Siegel cusp forms of the principal congruence subgroups of level $N \geq 3$ of any degree. A certain philosophical background of the theory in this paper is in [9], and some related development after 1997 can be found in [5] and [12] and their references, as well as in [8], [15], and [26].

The first version of this paper was written in 1996 and put on the Web as the preprint series 1997-37 of the Max-Planck-Institut für Mathematik. We intended to publish this after adding the conjectural dimension formula for the level 2 case, but this project has never been fulfilled. Since the 
second author passed away suddenly in 2010, the first author thinks that publication should not be delayed further and that a possible generalization should be given on another occasion. The first author would like to express his profound regret and sadness over the death of Hiroshi Saito.

\section{$\S 1$. Results on functional equations, poles, and residues}

Let $V_{n}$ be the vector space of $n \times n$ rational symmetric matrices. Let $\rho$ be the representation of $\mathrm{GL}_{n}(\mathbb{C})$ on $V_{n}(\mathbb{C})$ defined by $\rho(g) x=g x^{t} g$ for $x \in V_{n}(\mathbb{C})$ and $g \in \mathrm{GL}_{n}(\mathbb{C})$. Then $\left(\mathrm{GL}_{n}(\mathbb{C}), \rho, V_{n}(\mathbb{C})\right)$ is a prehomogeneous vector space whose set of singular points is $\left\{x \in V_{n}(\mathbb{C}) ; \operatorname{det}(S)=0\right\}$. The real orbits of $\{x \in V(\mathbb{R}) ; \operatorname{det}(S) \neq 0\}$ are given by $V_{n}^{i}(0 \leq i \leq n)$, where $V_{n}^{i}$ consists of real symmetric matrices with $i$ positive and $n-i$ negative eigenvalues. For any lattice $L \subset V$ invariant by $\Gamma=S L_{n}(\mathbb{Z}) \subset \mathrm{GL}_{n}(\mathbb{C})$, we put $L^{(i)}=L \cap V_{n}^{i}$ and denote by $L^{(i)} / \sim$ the set of $S L_{n}(\mathbb{Z})$ equivalence classes in $L^{(i)}$. In this paper, we consider the zeta functions of the prehomogeneous vector space $V_{n}$ defined for each $L, n$, and $i$ by

$$
\zeta_{i}(s, L)=c_{n} \sum_{x \in L^{i} / \sim} \frac{\mu(x)}{\operatorname{det}(x)^{s}}
$$

in the case $(n, i) \neq(2,1)$, where $\mu(x)$ is a certain volume of the stabilizer of $x$ and $c_{n}$ is a certain constant, or its modification in the case $(n, i)=(2,1)$. (For more precise definition, see [7] for the case $(n, i) \neq(2,1)$ and Section 5 for the case $(n, i)=(2,1)$.) In [7], an explicit formula for $\zeta_{i}(s, L)$ is given for $(n, i) \neq(2,1)$. In this section, we give results on functional equations between our zeta functions and their poles and residues. Here we mainly treat the case $n \geq 3$. The proof will be given in Section 4 . The case $n=1$ is the trivial case and will be omitted in this paper. The case $n=2$ is somewhat special but has been known by Shintani [21] and Sato [16]. This case will be treated separately in Section 5 from our standpoint with a new proof.

First, we recall the results from the previous paper (see $[7$, Part I]). Let $L_{n}$ be the set of $n \times n$ integral symmetric matrices, and let $L_{n}^{*}$ be the set of half integral symmetric matrices. For $L=L_{n}$ or $L_{n}^{*}$, our zeta function $\zeta_{i}(s, L)$ was originally defined for each $i$ with $0 \leq i \leq n$ by a certain infinite sum over elements of $L$ with signature $(i, n-1)$ as above. We have shown in [7] that $\zeta_{i}(s, L)$ depends only on $\delta=(-1)^{n-i}$ and $\epsilon=(-1)^{(n-i)(n-i+1) / 2}$, and we denote it by $\zeta(s, L, \delta, \epsilon)$ (for the precise definition, see [7]). We review the explicit formula for this zeta function. 
For the sake of simplicity, we put

$$
b_{n}=\frac{\left|\prod_{i=1}^{[(n-1) / 2]} B_{2 i}\right|}{2^{n-1}\left(\left[\frac{n-1}{2}\right]\right) !}
$$

where $B_{k}$ is the $k$ th Bernoulli number defined by $t e^{t} /\left(e^{t}-1\right)=\sum_{k=0}^{\infty} B_{k} t^{k} / k$ ! and $[x]$ is the greatest integer not more than $x$. When $n$ is even, we also use the notation

$$
B_{n / 2}^{\prime}=2\left(\frac{n}{2}\right) !(2 \pi)^{-n / 2} \zeta\left(\frac{n}{2}\right)(-1)^{1+n / 2}
$$

For odd $n$, we put

$$
\begin{aligned}
& Q_{n}(s)=\zeta\left(s-\frac{n-1}{2}\right) \prod_{i=1}^{(n-1) / 2} \zeta(2 s-(2 i-1)), \\
& R_{n}(s)=\zeta(s) \prod_{i=1}^{(n-1) / 2} \zeta(2 s-2 i) .
\end{aligned}
$$

If $n$ is odd with $n \geq 3$, by [7, Theorem 1.2], we have

$$
\begin{aligned}
& \zeta\left(s, L_{n}^{*}, \delta, \epsilon\right)=b_{n} 2^{(n-1) s}\left(Q_{n}(s)+\epsilon \delta^{(n+1) / 2}(-1)^{\left(n^{2}-1\right) / 8} R_{n}(s)\right), \\
& \zeta\left(s, L_{n}, \delta, \epsilon\right)=b_{n}\left(2^{(n-1) / 2} Q_{n}(s)+\epsilon \delta^{(n+1) / 2}(-1)^{\left(n^{2}-1\right) / 8} R_{n}(s)\right) .
\end{aligned}
$$

For even $n$, we put

$$
A_{n}(s)=\prod_{i=1}^{n / 2-1} \zeta(2 s-2 i) \quad \text { and } \quad B_{n}(s)=\prod_{i=1}^{n / 2} \zeta(2 s-(2 i-1)) .
$$

If $n$ is even with $n \geq 4$ or $(n, \delta)=(2,1)$, we also put

$$
\begin{aligned}
D_{n}^{*}(s, \delta)= & \frac{2(-1)^{[n / 4]} \Gamma(n / 2)}{(2 \pi)^{n / 2}} \\
& \times \sum_{(-1)^{n / 2} \delta d_{K}>0}\left|d_{K}\right|^{(n-1) / 2-s} L\left(\frac{n}{2}, \chi_{K}\right) \frac{\zeta(2 s) \zeta(2 s-n+1)}{L\left(2 s-\frac{n}{2}+1, \chi_{K}\right)},
\end{aligned}
$$

where $d_{K}$ runs through all the discriminants of quadratic fields $K$ or $\mathbb{Q} \oplus \mathbb{Q}$ such that $(-1)^{n / 2} \delta D_{K}>0$ and $\chi_{K}$ is the Dirichlet character associated with $K$. Let $D_{n}^{*}(s, \delta)=\sum_{d=1}^{\infty} H(n / 2, d, \delta) d^{-s}$. We define

$$
D(s, \delta)=\sum_{i=1}^{\infty} H\left(\frac{n}{2}, 4 d, \delta\right) d^{-s}
$$

By [7, Theorem 1.3], we have 


$$
\begin{aligned}
\zeta\left(s, L_{n}^{*}, \delta, \epsilon\right)= & b_{n} 2^{n s}\left((-1)^{[n / 4]} D_{n}^{*}(s, \delta) A_{n}(s)\right. \\
& \left.+\epsilon \delta_{n}(-1)^{n(n+2) / 8} \frac{2\left|B_{n / 2}^{\prime}\right|}{n} B_{n}(s)\right), \\
\zeta\left(s, L_{n}, \delta, \epsilon\right)= & b_{n}\left((-1)^{[n / 4]} D_{n}(s, \delta) A_{n}(s)\right. \\
& \left.+\epsilon \delta_{n}(-1)^{n(n+2) / 8} \frac{2^{(n+2) / 2}\left|B_{n / 2}^{\prime}\right|}{n} B_{n}(s)\right),
\end{aligned}
$$

where $\delta_{n}=1$ if $(-1)^{n / 2} \equiv \delta \bmod 4$ and where $\delta_{n}=0$ otherwise.

Using the notation of Shintani [21], for any $s \in \mathbb{C}$ and a positive integer $n$, we put

$$
\gamma_{n}(s)=\prod_{i=0}^{n-1} \Gamma\left(s+1+\frac{i}{2}\right)
$$

Now we state our results on functional equations and their poles and residues. Needless to say, we can recover Shintani's functional equation for $n \geq 3$ from our simpler results, Theorems 1 and 3 below.

When $n$ is odd with $n \geq 3$, then $\zeta(s, L, 1, \epsilon)=\zeta\left(s, L,-1,(-1)^{(n+1) / 2} \epsilon\right)$ for $L=L_{n}$ or $L_{n}^{*}$. So, it is enough to give a functional equation between $\zeta(s, L, 1,1)$ and $\zeta(s, L, 1,-1)$.

THEOREM 1. When $n$ is odd with $n \geq 3$, then

$$
\begin{aligned}
& \left(\begin{array}{c}
\zeta\left(\frac{n+1}{2}-s, L_{n}^{*}, 1,1\right) \\
\zeta\left(\frac{n+1}{2}-s, L_{n}^{*}, 1,-1\right)
\end{array}\right) \\
& =2^{-n s+\left(n^{2}-1\right) / 2} \pi^{-n s+n(n-1) / 4} \gamma_{n}\left(s-\frac{n+1}{2}\right) \\
& \quad \times\left(\begin{array}{c}
a(s)+b(s),-a(s)+b(s) \\
a(s)-b(s),-a(s)-b(s)
\end{array}\right)\left(\begin{array}{c}
\zeta\left(s, L_{n}, 1,1\right) \\
\zeta\left(s, L_{n}, 1,-1\right)
\end{array}\right),
\end{aligned}
$$

where

$$
a(s)=\left(\cos \frac{\pi s}{2}\right)(\cos \pi s)^{(n-1) / 2}
$$

and

$$
b(s)=(-1)^{\left(n^{2}-1\right) / 8}(\sin \pi s)^{(n-1) / 2} \times \begin{cases}\cos \frac{\pi s}{2} & \text { if } n \equiv 1 \bmod 4, \\ \sin \frac{\pi s}{2} & \text { if } n \equiv 3 \bmod 4 .\end{cases}
$$

Next, we give poles and residues of the above zeta functions. When $s_{0} \in \mathbb{C}$ is the possible simple pole of $\zeta\left(s, L_{n}^{*}, \delta, \epsilon\right)$ or $\zeta\left(s, L_{n}, \delta, \epsilon\right)$, we denote by $r_{n, \delta, \epsilon}\left(s_{0}\right)$ or $r_{n, \delta, \epsilon}^{*}\left(s_{0}\right)$, respectively, the residue of each zeta function at $s_{0}$. 
We understand that this value is zero when the zeta function is holomorphic at $s_{0}$. When $n$ is odd, we can determine the poles and residues exactly, as shown in the following theorem.

THEOREM 2. We assume that $n \geq 3$ is an odd integer, and we define $b_{n}$ as before. Then, the zeta function $\zeta\left(s, L_{n}^{*}, \delta, \epsilon\right)$ or $\zeta\left(s, L_{n}, \delta, \epsilon\right)$ has possible poles only at $s=l / 2$, where $l$ is an integer with $2 \leq l \leq n+1$. The residues are given as follows.

(1) When $n=3$, we have

$$
\begin{aligned}
& r_{3, \delta, \epsilon}^{*}(1)=(2 \epsilon-1) b_{3}, \\
& r_{3, \delta, \epsilon}(1)=(\epsilon-1) b_{3} / 2 .
\end{aligned}
$$

In particular, we have $r_{3, \delta, 1}(1)=0$ always. When $n>3$, we have $r_{n, \delta, \epsilon}^{*}(1)=$ $r_{n, \delta, \epsilon}(1)=0$, and the zeta functions are holomorphic at 1 .

(2) For each integer $j$ with $2 \leq j \leq(n-1) / 2$, we have

$$
\begin{aligned}
& r_{n, \delta, \epsilon}^{*}(j)=2^{(n-1) j-1} b_{n} \zeta(j-(n-1) / 2) \prod_{i=1, i \neq j}^{(n-1) / 2} \zeta(2 j-2 i+1), \\
& r_{n, \delta, \epsilon}(j)=2^{(n-3) / 2} b_{n} \zeta(j-(n-1) / 2) \prod_{i=1, i \neq j}^{(n-1) / 2} \zeta(2 j-2 i+1) .
\end{aligned}
$$

In particular, if $j<(n-1) / 2$ and $j \equiv(n-1) / 2 \bmod 2$, then the zeta functions are holomorphic at $s=j$.

(3) We have

$$
\begin{aligned}
& r_{n, \delta, \epsilon}^{*}((n+1) / 2)=2^{\left(n^{2}-1\right) / 2} b_{n} \prod_{i=1}^{(n-1) / 2} \zeta(n-2 i+2), \\
& r_{n, \delta, \epsilon}((n+1) / 2)=2^{(n-1) / 2} b_{n} \prod_{i=1}^{(n-1) / 2} \zeta(n-2 i+2) .
\end{aligned}
$$

(4) For each integer $j$ with $1 \leq j \leq(n-1) / 2$, we have

$$
\begin{aligned}
r_{n, \delta, \epsilon}^{*}\left(j+\frac{1}{2}\right)= & \epsilon 2^{(n-1)(j+1 / 2)} \delta^{(n+1) / 2}(-1)^{\left(n^{2}-1\right) / 8} \\
& \times b_{n} \zeta\left(j+\frac{1}{2}\right) \prod_{i=1}^{(n-1) / 2} \zeta(2 j-2 i+1),
\end{aligned}
$$




$$
r_{n, \delta, \epsilon}\left(j+\frac{1}{2}\right)=\epsilon \delta^{(n+1) / 2}(-1)^{\left(n^{2}-1\right) / 8} b_{n} \zeta\left(j+\frac{1}{2}\right) \prod_{i=1}^{(n-1) / 2} \zeta(2 j-2 i+1)
$$

When $n$ is even with $n \geq 4$, then $\zeta(s, L, 1,1)=\zeta(s, L, 1,-1)$ if $n \equiv$ $2 \bmod 4$, and $\zeta(s, L,-1,1)=\zeta(s, L,-1,-1)$ if $n \equiv 0 \bmod 4$. We give the functional equation for each case.

TheOREM 3. We put $c(s)=(\cos \pi s)^{n / 2-1} \sin \pi s$. When $n \equiv 0 \bmod 4$, we have

$$
\begin{aligned}
& \left(\begin{array}{c}
\zeta\left(\frac{n+1}{2}-s, L_{n}^{*}, 1,1\right) \\
\zeta\left(\frac{n+1}{2}-s, L_{n}^{*}, 1,-1\right) \\
\zeta\left(\frac{n+1}{2}-s, L_{n}^{*},-1,1\right)
\end{array}\right) \\
& =2^{-n s+n^{2} / 2-1} \pi^{-n s+n(n-1) / 4} \gamma_{n}\left(s-\frac{n+1}{2}\right) \\
& \quad \times\left(\begin{array}{rr}
(\cos \pi s)^{n / 2}+(-1)^{n(n-2) / 8}(\sin \pi s)^{n / 2}(\cos \pi s)^{n / 2}-(-1)^{n(n-2) / 8}(\sin \pi s)^{n / 2} & 0 \\
(\cos \pi s)^{n / 2}-(-1)^{n(n-2) / 8}(\sin \pi s)^{n / 2}(\cos \pi s)^{n / 2}+(-1)^{n(n-2) / 8}(\sin \pi s)^{n / 2} & 0 \\
(\cos \pi s)^{n / 2-1} & -2 c(s)
\end{array}\right) \\
& \quad \times\left(\begin{array}{r}
\zeta\left(s, L_{n}, 1,1\right) \\
\zeta\left(s, L_{n}, 1,-1\right) \\
\zeta\left(s, L_{n},-1,1\right)
\end{array}\right) .
\end{aligned}
$$

When $n \equiv 2 \bmod 4$ with $n \geq 6$, we have

$$
\begin{aligned}
& \left(\begin{array}{c}
\zeta\left(\frac{n+1}{2}-s, L_{n}^{*}, 1,1\right) \\
\zeta\left(\frac{n+1}{2}-s, L_{n}^{*},-1,1\right) \\
\zeta\left(\frac{n+1}{2}-s, L_{n}^{*},-1,-1\right)
\end{array}\right) \\
& =2^{-n s+n^{2} / 2-1} \pi^{-n s+n(n-1) / 4} \gamma_{n}\left(s-\frac{n+1}{2}\right) \\
& \quad \times\left(\begin{array}{c}
2(\cos \pi s)^{n / 2} \\
2(\cos \pi s)^{n / 2-1} c(s)+(-1)^{n(n-2) / 8}(\sin \pi s)^{n / 2} c(s)-(-1)^{n(n-2) / 8}(\sin \pi s)^{n / 2} \\
2(\cos \pi s)^{n / 2-1} c(s)-(-1)^{n(n-2) / 8}(\sin \pi s)^{n / 2} c(s)+(-1)^{n(n-2) / 8}(\sin \pi s)^{n / 2}
\end{array}\right) \\
& \quad \times\left(\begin{array}{c}
\zeta\left(s, L_{n}, 1,1\right) \\
\zeta\left(s, L_{n},-1,1\right) \\
\zeta\left(s, L_{n},-1,-1\right)
\end{array}\right) .
\end{aligned}
$$

Now, we give locations of poles and their residues for even $n$. 
TheOREM 4. We assume that $n \geq 4$ is an even integer. Then, the zeta function $\zeta\left(s, L_{n}^{*}, \delta, \epsilon\right)$ or $\zeta\left(s, L_{n}, \delta, \epsilon\right)$ has possible poles only at $s=l / 2$, where $l$ is an integer with $1 \leq l \leq n+1$. The residues are given as follows.

(1) Unless $(n, \delta)=(4,-1)$, we have

$$
\begin{aligned}
& r_{n, \delta, \epsilon}^{*}(1)=2^{n} b_{n} \epsilon \delta_{n}(-1)^{n(n+2) / 8} \frac{\left|B_{n^{\prime} / 2}\right|}{n} \prod_{i=2}^{n / 2} \zeta(3-2 i), \\
& r_{n, \delta, \epsilon}(1)=2^{-n / 2} r_{n, \delta, \epsilon}^{*}(1),
\end{aligned}
$$

and if $(n, \delta)=(4,-1)$, we have

$$
r_{4,-1, \epsilon}^{*}(1)=r_{4,-1, \epsilon}(1)=12 \cdot \pi^{-4} \zeta(3) .
$$

(2) For each integer $j$ with $2 \leq j \leq n / 2$, we have

$$
\begin{aligned}
& r_{n, \delta, \epsilon}^{*}(j)=2^{n j} b_{n} \epsilon \delta_{n}(-1)^{n(n+2) / 8} \frac{\left|B_{n^{\prime} / 2}\right|}{n} \prod_{i=1, i \neq j}^{n / 2} \zeta(2 j-2 i+1), \\
& r_{n, \delta, \epsilon}(j)=2^{n(1 / 2-j)} r_{n, \delta, \epsilon}^{*}(j) .
\end{aligned}
$$

In particular, if $(-1)^{n / 2} \equiv-\delta \bmod 4$, then the zeta functions are holomorphic at $s=j$.

(3) We have

$$
\begin{aligned}
& r_{n, \delta, \epsilon}^{*}((n+1) / 2)=2^{n^{2} / 2} b_{n} \pi^{-n / 2} \Gamma(n / 2) \zeta(n) \prod_{i=1}^{n / 2-1} \zeta(n+1-2 i), \\
& r_{n, \delta, \epsilon}((n+1) / 2)=2^{n^{2} / 2} r_{n, \delta, \epsilon}^{*}((n+1) / 2) .
\end{aligned}
$$

(4) For each integer $j$ with $1 \leq j \leq n / 2-1$, we have

$$
\begin{aligned}
& r_{n, \delta, \epsilon}^{*}(j+1 / 2)=2^{n(j+1 / 2)-1}(-1)^{[n / 4]} b_{n} D_{n}^{*}(j+1 / 2, \delta) \prod_{i=1, i \neq j}^{n / 2-1} \zeta(2 i+1-2 j), \\
& r_{n, \delta, \epsilon}(j+1 / 2)=(-1)^{[n / 4]} b_{n} D_{n}(j+1 / 2, \delta) \prod_{i=1, i \neq j}^{n / 2-1} \zeta(2 i+1-2 j) .
\end{aligned}
$$

REMARK. We do not know whether $D_{n}^{*}(j+1 / 2, \delta)$ or $D_{n}(j+1 / 2, \delta)$ vanishes or not. 


\section{§2. Fourier coefficients of Eisenstein series}

In order to prove the results in Section 1 directly, we use the theory of real analytic Eisenstein series. In this section, we review several results on Fourier coefficients of some real analytic or holomorphic Eisenstein series of halfintegral weight of one variable. Our main results here are Proposition 2.2 and Corollary 2.4. The Fourier coefficients of such series are investigated by, among others, Shimura [20], Siegel [22], Cohen [3], Zagier [27], Sturm [23], and Goldfeld and Hoffstein [4]. Although we need some modification of their results, the structure of the proof is essentially due to them. We will sometimes sketch the proof for the convenience of the readers. For any $\sigma, z \in \mathbb{C}$ with $z \neq 0$, we define $z^{\sigma}=e^{\sigma \log (z)}$, where $\arg (z)$ is taken so that $-\pi<\arg (z) \leq \pi$. For the sake of simplicity, we write $e(z)=e^{2 \pi i z}$. We denote the upper half-plane by $H=\{z \in \mathbb{C} ; \operatorname{Im}(z)>0\}$, and we write $z=x+i y$ for any $z \in H$. For each $\sigma \in \mathbb{C}$, each odd (positive or negative) integer $k$, and $z \in H$, define the Eisenstein series $E(k, \sigma, z)$ by

$$
E(k, \sigma, z)=y^{\frac{\sigma}{2}} \sum_{d=1, \text { odd }}^{\infty} \sum_{c=-\infty}^{\infty}\left(\frac{4 c}{d}\right) \epsilon_{d}^{-k}(4 c z+d)^{(k / 2)}|4 c z+d|^{-\sigma},
$$

where $(* / *)$ is the quadratic residue symbol whose precise meaning is as given in Shimura [19, p. 442], and $\epsilon_{d}=1$ or $i$, if $d \equiv 1$ or $3 \bmod 4$, respectively. When $-k+2 \sigma-4>0$, this series converges absolutely and uniformly. We also define $E^{*}(k, \sigma, z)$ by

$$
\begin{aligned}
& E^{*}(k, \sigma, z) \\
& \quad=E\left(-\frac{1}{4 z}\right)(-2 i z)^{(k / 2)} \\
& \quad=y^{\frac{\sigma}{2}} 2^{(k / 2)-\sigma} e\left(-\frac{k}{8}\right) \sum_{d=1, \text { odd }}^{\infty} \sum_{b=-\infty}^{\infty}\left(\frac{-b}{d}\right) \epsilon_{d}^{-k}(d z+b)^{(k / 2)}|d z+b|^{-\sigma} .
\end{aligned}
$$

As usual, we write

$$
\Gamma_{0}(4)=\left\{g=\left(\begin{array}{ll}
a & b \\
c & d
\end{array}\right) \in S L_{2}(\mathbb{Z}) ; c \equiv 0 \bmod 4\right\} .
$$

For any element $\gamma=\left(\begin{array}{ll}a & b \\ c & d\end{array}\right) \in \Gamma_{0}(4)$, put $j(\gamma, z)=\theta(\gamma z) / \theta(z)$, where $\theta(z)=$ $\sum_{n \in \mathbb{Z}} e\left(n^{2} z\right)$. It is known that

$$
j(\gamma, z)=\epsilon_{d}^{-1}\left(\frac{c}{d}\right)(c z+d)^{1 / 2} .
$$

We have 


$$
\begin{gathered}
E(k, \sigma, \gamma z)=j(\gamma, z)^{-k} E(k, \sigma, z), \\
E^{*}(k, \sigma, \gamma z)=j(\gamma, z)^{-k} E^{*}(k, \sigma, z),
\end{gathered}
$$

where we write $\gamma z=(a z+b)(c z+d)^{-1}$. So, these functions behave like modular forms of weight $-k / 2$, though they are not holomorphic if $\sigma \neq 0$. For odd $k<-5$, two holomorphic functions $E(-k, 0, z)$ and $E^{*}(-k, 0, z)$ form a basis of the orthogonal complement of cusp forms of weight $-k / 2$ in modular forms of weight $-k / 2$ belonging to $\Gamma_{0}(4)$ (see [19]). After Siegel and Shimura, we define a confluent hypergeometric function $W(y, \alpha, \beta)$ by

$$
W(y, \alpha, \beta)=\int_{0}^{\infty}(u+1)^{\alpha-1} u^{\beta-1} e^{-y u} d u,
$$

where $y>0$ and $\alpha, \beta \in \mathbb{C}$. This converges for $\operatorname{Re}(\beta)>0$ and meromorphically continues to the whole $(\alpha, \beta) \in \mathbb{C}^{2}$. For any integer $d$ and $\alpha, \beta \in \mathbb{C}$ with $\operatorname{Re}(\alpha)>0, \operatorname{Re}(\beta)>0, \operatorname{Re}(\alpha+\beta)>1$, we also define $\tau_{d}(y, \alpha, \beta)$ by

$$
\begin{aligned}
& i^{\alpha-\beta}(2 \pi)^{-\alpha-\beta} \Gamma(\alpha) \Gamma(\beta) \tau_{d}(y, \alpha, \beta) \\
& \quad= \begin{cases}d^{\alpha+\beta-1} e^{-2 \pi d y} W(4 \pi d y, \alpha, \beta) & (d>0), \\
|d|^{\alpha+\beta-1} e^{-2 \pi|d| y} W(4 \pi|d| y, \beta, \alpha) & (d<0), \\
\Gamma(\alpha+\beta-1)(4 \pi y)^{1-\alpha-\beta} & (d=0) .\end{cases}
\end{aligned}
$$

This is also meromorphically continued. The Fourier expansions of $E(k, \sigma, z)$ and $E^{*}(k, \sigma, z)$ are of the following form:

$$
\begin{aligned}
y^{-\sigma / 2} E(k, \sigma, z) & =1+\sum_{d=-\infty}^{\infty} \beta(d, \sigma, k) e(d x) \tau_{d}\left(y, \frac{\sigma-k}{2}, \frac{\sigma}{2}\right), \\
y^{-\sigma / 2} 2^{\sigma-k / 2} e\left(\frac{k}{8}\right) E^{*}(k, \sigma, z) & =\sum_{d=-\infty}^{\infty} \alpha(d, \sigma, k) e(d x) \tau_{d}\left(y, \frac{\sigma-k}{2}, \frac{\sigma}{2}\right),
\end{aligned}
$$

where we put $z=x+i y(x, y \in \mathbb{R})$ and

$$
\begin{aligned}
& \alpha(d, \sigma, k)=\sum_{c=1}^{\infty}\left(\frac{-4}{c}\right) \epsilon_{c}^{-k} c^{k / 2-\sigma} \sum_{m=1}^{c}\left(\frac{m}{c}\right) e(d m / c), \\
& \beta(d, \sigma, k)=\sum_{c=1}^{\infty}(4 c)^{k / 2-\sigma} \sum_{m=1}^{4 c} \epsilon_{m}^{-k}\left(\frac{4 c}{m}\right) e^{2 \pi i m d / 4 c} .
\end{aligned}
$$


To obtain Fourier coefficients suitable for our purpose, we put

$$
F(k, \sigma, z)=E(k, \sigma, z)+2^{k / 2-\sigma}(e(k / 8)+e(-k / 8)) E^{*}(k, \sigma, z) .
$$

This kind of linear combination has already been considered by Cohen [3], Zagier [27], and Sturm [23] for $\sigma=0$. We need the coefficients also for $\sigma \neq 0$, which was calculated in Goldfeld and Hoffstein [4]. Since we need slightly different details here, we review this. We write the Fourier expansion of $F(k, \sigma, z)$ as

$$
\begin{aligned}
y^{-\sigma / 2} F(k, \sigma, z) & =\sum_{d=-\infty}^{\infty} c_{d}(y) e(d x) \\
& =1+\sum_{d=-\infty}^{\infty} C(d, \sigma, k) e(d x) \tau_{d}\left(y, \frac{\sigma-k}{2}, \frac{\sigma}{2}\right),
\end{aligned}
$$

where each $c_{d}(y)$ is the Fourier coefficient depending on $y$ and $d$ and each $C(d, \sigma, k)$ is a constant which does not depend on $z$. Following the method of Shimura [20] and Sturm [23], we can calculate $C(d, \sigma, k)$ explicitly. For the convenience of the readers, we sketch the calculation here. By definition,

$$
C(d, \sigma, k)=\beta(d, \sigma, k)+2^{k-2 \sigma}\left(1+i^{-k}\right) \alpha(d, \sigma, k) .
$$

By Sturm [23, Lemma 2, arguments on p. 228], we have

$$
\begin{aligned}
C(d, \sigma, k) & =\alpha(d, \sigma, k)\left(2^{k-2 \sigma}\left(1+i^{-k}\right)+\sum_{r=2}^{\infty} \sum_{l=1}^{2^{r}} \epsilon_{l}^{-k}\left(\frac{2^{r}}{l}\right) e\left(\frac{d l}{2^{r}}\right) 2^{(k / 2-\sigma) r}\right) \\
& =\alpha(d, \sigma, k) A(d, \sigma, k),
\end{aligned}
$$

where

$$
\begin{aligned}
A(d, \sigma, k)= & 2^{k-2 \sigma}\left(1+i^{-k}\right) \\
& +\sum_{r=2}^{\infty} \frac{1}{2}\left(\left(1+i^{-k}\right) U_{d}(r)+\left(1-i^{-k}\right) V_{d}(r)\right) 2^{(k / 2-\sigma) r}, \\
U_{d}(r)= & \sum_{l=1}^{2^{r}}\left(\frac{2^{r}}{l}\right) e\left(\frac{d l}{2^{r}}\right) \\
V_{d}(r)= & \sum_{l=1}^{2^{r}}\left(\frac{-2^{r}}{l}\right) e\left(\frac{d l}{2^{r}}\right) .
\end{aligned}
$$


Assume that $d \neq 0$, and put $d=2^{t} d_{0}$, where $d_{0}$ is an odd (positive or negative) integer and $t$ is a nonnegative integer. If $t$ is even, then we see that $U_{d}(2 i)=2^{2 i-1}$ for $1 \leq i \leq t / 2, U_{d}(t+3)=\left(2 / d_{0}\right) 2^{t+3 / 2}$, and $U_{d}(r)=0$ in the remaining cases and that $V_{d}(t+2)=2^{t+1} i\left(-1 / d_{0}\right), V_{d}(t+3)=2^{t+3 / 2}\left(-2 / d_{0}\right)$, and $V_{d}(r)=0$ for the other $r$. If $t$ is odd, then $V_{d}(r)=0$ for all $r$, and $U_{d}(2 i)=2^{2 i-1}$ for $1 \leq i \leq(t-1) / 2, U_{d}(t+1)=-2^{t}$, and $U_{d}(r)=0$ for the other $r$. Hence, if $t$ is even, then we have

$$
\begin{aligned}
& \sum_{r=2}^{\infty} \frac{1}{2}\left(1+i^{-k}\right) U_{d}(r) 2^{(k / 2-\sigma) r} \\
& \quad=\left(1+i^{-k}\right)\left(\frac{2^{k-2 \sigma}\left(1-2^{(k / 2-\sigma+1) t}\right)}{1-2^{k-2 \sigma+2}}+2^{3(k / 2-\sigma)+1 / 2+(k / 2-\sigma+1) t}\left(\frac{2}{d_{0}}\right)\right), \\
& \sum_{r=2}^{\infty} \frac{1}{2}\left(1-i^{-k}\right) V_{d}(r) 2^{(k / 2-\sigma) r} \\
& =(-1)^{(k+1) / 2}\left(1+i^{-k}\right) \\
& \quad \times\left(2^{3(k / 2-\sigma)+1 / 2} \cdot 2^{(k / 2-\sigma+1) t}\left(\frac{-2}{d_{0}}\right)+2^{k-2 \sigma+(k / 2-\sigma+1) t}\left(\frac{-1}{d_{0}}\right)\right),
\end{aligned}
$$

and if $t$ is odd, then we have

$$
\begin{aligned}
& \sum_{r=2}^{\infty} \frac{1}{2}\left(1+i^{-k}\right) U_{d}(r) 2^{(k / 2-\sigma) r} \\
& =\left(1+i^{-k}\right)\left(-2^{k / 2-\sigma-1} \cdot 2^{(k / 2-\sigma+1) t}+\frac{2^{k-2 \sigma}\left(1-2^{(k-2 \sigma+2)(t-1) / 2}\right)}{1-2^{k-2 \sigma+2}}\right), \\
& \quad \sum_{r=2}^{\infty} \frac{1}{2}\left(1-i^{-k}\right) V_{d}(r) 2^{(k / 2-\sigma) r}=0 .
\end{aligned}
$$

We have $(-1)^{(k+1) / 2}\left(-1 / d_{0}\right)=1$ or -1 , if $d_{0} \equiv(-1)^{(k+1) / 2} \bmod 4$ or not, respectively. Hence we have the following.

LEMMA 2.1.

(1) If $d_{0} \equiv(-1)^{(k+1) / 2} \bmod 4$, then we have

$$
\begin{aligned}
& \sum_{t=0}^{\infty} A\left(d_{0} 2^{2 t}, \sigma, k\right) 2^{2 t(\sigma-k / 2-1-s)} \\
& \quad=\left(1+i^{-k}\right) 2^{k-2 \sigma+1} \times \frac{\left(1+2^{k / 2-\sigma+1 / 2}\left(\frac{2}{d_{0}}\right)\right)\left(1-2^{\sigma-k / 2-3 / 2-2 s}\left(\frac{2}{d_{0}}\right)\right)}{\left(1-2^{-2 s}\right)\left(1-2^{2 \sigma-k-2-2 s}\right)} .
\end{aligned}
$$


(2) If $d_{0} \not \equiv(-1)^{(k+1) / 2} \bmod 4$, then we have

$$
\sum_{t=0}^{\infty} A\left(d_{0} 2^{2 t}, \sigma, k\right) 2^{2 t(\sigma-k / 2-1-s)}=\left(1+i^{-k}\right) \frac{2^{-1-2 s}\left(1-2^{k-2 \sigma+1}\right)}{\left(1-2^{-2 s}\right)\left(1-2^{2 \sigma-k-2-2 s}\right)} .
$$

(3) For any odd $d_{0}$, we have

$$
\begin{aligned}
& \sum_{t=0}^{\infty} A\left(d_{0} 2^{2 t+1}, \sigma, k\right) 2^{(2 t+1)(\sigma-k / 2-1-s)} \\
& \quad=\left(1+i^{-k}\right) \frac{2^{\sigma-k / 2-2-3 s}\left(1-2^{k-2 \sigma+1}\right)}{\left(1-2^{-2 s}\right)\left(1-2^{2 \sigma-k-2-2 s}\right)} .
\end{aligned}
$$

By Shimura [20, Proposition 2], for any square-free integer $m$, we have

$$
\begin{aligned}
& \sum_{f=1}^{\infty} \alpha\left(m f^{2}, \sigma, k\right) f^{2 \sigma-k-2-2 s} \\
& \quad=\frac{L_{2}\left(\sigma-(k+1) / 2, \omega_{1}\right) \zeta_{2}(2 s) \zeta(2 s-2 \sigma+k+2)}{\zeta_{2}(2 \sigma-k-1) L_{2}\left(2 s-\sigma+(k+3) / 2, \omega_{1}\right)}
\end{aligned}
$$

where we denote by $\zeta_{2}$ or $L_{2}$ the usual zeta or $L$ functions such that their Euler 2-factors are omitted and where $\omega_{1}$ is the primitive character defined by

$$
\omega_{1}(a)=\left(\frac{-1}{a}\right)^{(k+1) / 2}\left(\frac{4 m}{a}\right)
$$

for $(a, 4 m)=1$. Here $(* / *)$ is the quadratic residue symbol defined in Shimura [19]. Now, for any square-free integer $m$, we calculate the Dirichlet series

$$
\sum_{f=1}^{\infty} e\left(\frac{k}{8}\right) C\left(m f^{2}, \sigma, k\right)\left(|m| f^{2}\right)^{\sigma-k / 2-1-s}
$$

By the above formulas (2) and (3) in Lemma 2.1, if $f$ is odd, then we have $C\left(m f^{2}, \sigma, k\right)=0$ unless $m$ is odd and $m \equiv(-1)^{(k+1) / 2} \bmod 4$. Hence, we can assume that $d_{K} f_{1}^{2}=(-1)^{(k+1) / 2} m f^{2}$ for some integer $f_{1}$ and the fundamental discriminant $d_{K}$ of the quadratic field $K=\mathbb{Q}\left(\sqrt{(-1)^{(k+1) / 2} m}\right)$ or $d_{K}=1$ for $m=(-1)^{(k+1) / 2}$. Now we are ready to prove the following. 
Proposition 2.2. We have

$$
\begin{aligned}
\sum_{f=1}^{\infty} e\left(\frac{k}{8}\right) C\left((-1)^{(k+1) / 2} d_{K} f^{2}, \sigma, k\right)\left(\left|d_{K}\right| f^{2}\right)^{\sigma-k / 2-1-s} \\
=(-1)^{\left(k^{2}-1\right) / 8} 2^{k-2 \sigma+3 / 2} \frac{\left|d_{K}\right|^{\sigma-k / 2-1}}{\left|d_{K}\right|^{s}} \\
\quad \times \frac{L\left(\sigma-(k+1) / 2, \chi_{K}\right) \zeta(2 s) \zeta(2 s-2 \sigma+k+2)}{\zeta(2 \sigma-k-1) L\left(2 s-\sigma+(k+3) / 2, \chi_{K}\right)}
\end{aligned}
$$

where $\chi_{K}$ is the Dirichlet character associated with $K$ and regarded as the trivial character if $d_{K}=1$.

Proof. This can be shown by calculating a kind of convolution product of the Dirichlet series in Lemma 2.1 and Shimura's results, noting that $e(k / 8)\left(1+i^{-k}\right)=e(k / 8)+e(-k / 8)=\sqrt{2}(-1)^{\left(k^{2}-1\right) / 8}$.

Proposition 2.3. If $\sigma>k / 2+1$, then we have

$$
C(0, \sigma, k)=2^{k-2 \sigma+1}\left(1+i^{-k}\right) \frac{\zeta(2 \sigma-k-2)}{\zeta(2 \sigma-k-1)} .
$$

Proof. When $d=0$, we have $V(r)=0$ for all $r$, and $U(r)=2^{r-1}$ or 0 for $r$ even or odd, respectively. Since

$$
\sum_{r=1}^{\infty} U(2 r) 2^{(k / 2-\sigma) 2 r}=\frac{2^{k-2 \sigma+1}}{1-2^{k-2 \sigma+2}},
$$

we have

$$
A(0, \sigma, k)=2^{k-2 \sigma+1}\left(1+i^{-k}\right) \frac{1-2^{k-2 \sigma+1}}{1-2^{k-2 \sigma+2}} .
$$

On the other hand, by Shimura [20, p. 89, (3.9)], we have

$$
\alpha(0, \sigma, k)=\frac{\zeta_{2}(2 \sigma-k-2)}{\zeta_{2}(2 \sigma-k-1)} .
$$

Hence, we have our results.

Corollary 2.4. We have

$$
\begin{aligned}
c_{0}(y)=1 & +2^{3 k / 2-3 \sigma+7 / 2}(-1)^{\left(k^{2}-1\right) / 8} \\
& \times \pi \frac{\Gamma(\sigma-k / 2-1) \zeta(2 \sigma-k-2)}{\Gamma((\sigma-k) / 2) \Gamma(\sigma / 2) \zeta(2 \sigma-k-1)} y^{1-\sigma+k / 2} .
\end{aligned}
$$




\section{§3. Dirichlet series associated to Eisenstein series}

\section{1 .}

First, we prove functional equations between $D_{n}^{*}((n+1) / 2-s, \delta)$ and $D_{n}(s, \delta)$ only for even $n \geq 4$ with $\delta= \pm 1$ or $(n, \delta)=(2,1)$. The case $(n, \delta)=$ $(2,-1)$ is exceptional, so we exclude this case for the moment and treat it next in Section 5. Our proof is based on the usual argument by Hecke on functional equations of Mellin transforms of automorphic forms. This type of functional equation has been more or less calculated by Goldfeld and Hoffstein [4] for a different purpose, but the modular forms in question are slightly different, and we need some new calculations here. For any function $f(z)$ on $H$, we define two operators $\left.f\right|_{k} W_{4}$ and $f \mid U_{4}$ by

$$
\begin{aligned}
\left(\left.f\right|_{k} W_{4}\right)(z) & =f(-1 / 4 z)(-2 i z)^{k / 2} \\
\left(f \mid U_{4}\right)(z) & =\frac{1}{4} \sum_{\nu=0}^{3} f\left(\frac{z+\nu}{4}\right) .
\end{aligned}
$$

We define $F(z)=F(k, \sigma, z)$ as in Section 2 .

LEMMA 3.1. We have the following relation:

$$
\left.F\right|_{k} W_{4}=2^{(k+1) / 2}(-1)^{\left(k^{2}-1\right) / 8} F \mid U_{4} .
$$

Proof. This can be proved in the same way as in Kohnen [10], and we sketch the outline. Put

$$
g_{1}(z)=\frac{1}{4}\left(F\left(\frac{z+1}{4}\right)+F\left(\frac{z+3}{4}\right)\right)
$$

and

$$
g_{2}(z)=\frac{1}{4}\left(F\left(\frac{z}{4}\right)+F\left(\frac{z+2}{4}\right)\right) .
$$

Then, $F \mid U_{4}=g_{1}+g_{2}$. As was shown in Section 2, the coefficients of $F(z)$ of $e(d x)$ are 0 , unless $d \equiv 0$ or $(-1)^{(k+1) / 2} \bmod 4$. Since $e(d / 2)+1=e(d / 4)+$ $e(3 d / 4)$ if $d \equiv 0 \bmod 4$ or $d \equiv 1 \bmod 2$, we have $g_{1}=g_{2}$. Hence, using the same argument as in Kohnen [10, p. 255], we have 


$$
\begin{aligned}
F\left|U_{4}\right|_{k} W_{4} & =\left.2 g_{1}\right|_{k} W_{4} \\
& =2^{-k / 2-1}\left(F\left(z-\frac{1}{4}\right) e(-k / 8)+F\left(z+\frac{1}{4}\right) e(k / 8)\right) .
\end{aligned}
$$

It is easy to see that $e(-d / 4) e(-k / 8)+e(d / 4) e(k / 8)=\sqrt{2}(-1)^{\left(k^{2}-1\right) / 8}$ if $d \equiv$ 0 or $(-1)^{(k+1) / 2} \bmod 4$. Hence, $F\left|U_{4}\right|_{k} W_{4}=2^{-(k+1) / 2}(-1)^{\left(k^{2}-1\right) / 8} F$, and since $W_{4}^{2}$ acts trivially on $F$ by Shimura [18, p. 448], we prove our lemma.

Now, we put $g(z)=y^{-\sigma / 2} F(k, \sigma, z)$ and $h(z)=\left(y^{-\sigma / 2} F(k, \sigma, z)\right) \mid U_{4}$. We write the Fourier expansion of $g(z)$ as

$$
g(z)=\sum_{d=-\infty}^{\infty} c_{d}(y) e(d x) .
$$

Then we have

$$
h(z)=\sum_{d=-\infty}^{\infty} c_{4 d}(y / 4) e(d x) .
$$

Put $G(y)=g(i y)$, and put $H(y)=h(i y)$. Then, by the above lemma, we have $G(1 / 4 y)=(-1)^{\left(k^{2}-1\right) / 8} \sqrt{2} y^{\sigma-k / 2} H(y)$ and $G(1 / 2 y)=C_{k} y^{\sigma-k / 2} H(y / 2)$, where $C_{k}=2^{(k+1) / 2-\sigma}(-1)^{\left(k^{2}-1\right) / 8}$. Define two Dirichlet series by

$$
\begin{aligned}
& \Phi_{\sigma, k}(s)=\int_{0}^{\infty}\left(G(y)-c_{0}(y)\right) y^{s-1} d y \\
& \Psi_{\sigma, k}(s)=\int_{0}^{\infty}\left(H(y)-c_{0}(y / 4)\right) y^{s-1} d y
\end{aligned}
$$

Then, by the usual argument of Hecke, we have

$$
\begin{aligned}
2^{s} \Phi_{\sigma, k}(s)= & \int_{0}^{\infty}\left(G\left(\frac{y}{2}\right)-c_{0}\left(\frac{y}{2}\right)\right) y^{s-1} d y \\
= & \int_{1}^{\infty}\left(G\left(\frac{y}{2}\right)-c_{0}\left(\frac{y}{2}\right)\right) y^{s-1} d y+\int_{0}^{1}\left(G\left(\frac{y}{2}\right)-c_{0}\left(\frac{y}{2}\right)\right) y^{s-1} d y \\
= & \int_{1}^{\infty}\left(G\left(\frac{y}{2}\right)-c_{0}\left(\frac{y}{2}\right)\right) y^{s-1} d y \\
& +\int_{1}^{\infty}\left(C_{k} H\left(\frac{y}{2}\right) y^{\sigma-k / 2}-c_{0}\left(\frac{1}{2 y}\right)\right) y^{-s-1} d y
\end{aligned}
$$




$$
\begin{aligned}
= & \int_{1}^{\infty}\left(G\left(\frac{y}{2}\right)-c_{0}\left(\frac{y}{2}\right)\right) y^{s-1} d y \\
& +C_{k} \int_{1}^{\infty}\left(H\left(\frac{y}{2}\right)-c_{0}\left(\frac{y}{8}\right)\right) y^{\sigma-k / 2-s-1} d y \\
& -\int_{1}^{\infty} c_{0}\left(\frac{1}{2 y}\right) y^{-s-1} d y+C_{k} \int_{1}^{\infty} c_{0}\left(\frac{y}{8}\right) y^{\sigma-k / 2-s-1} d y .
\end{aligned}
$$

We have $c_{0}(y)=1+c(\sigma) y^{1-\sigma+k / 2}$ for a certain function of $c(\sigma)$ of $\sigma$ which does not depend on $y$. (The exact value is given in Corollary 2.4.) So, if $s>\sigma-k / 2$ and $s>1$, then we have

$$
\begin{aligned}
2^{s} \Phi_{\sigma, k}(s)+\int_{1}^{\infty} c_{0}\left(\frac{1}{2 y}\right) y^{-s-1} d y-C_{k} \int_{1}^{\infty} c_{0}\left(\frac{y}{8}\right) y^{\sigma-k / 2-s-1} d y \\
=2^{s} \Phi_{\sigma, k}(s)+\frac{c(\sigma) 2^{\sigma-k / 2-1}}{s-\sigma+k / 2+1} \\
\quad+C_{k} \frac{c(\sigma) 8^{\sigma-k / 2-1}}{1-s}+\frac{1}{s}+C_{k} \frac{1}{\sigma-k / 2-s} \\
=\int_{1}^{\infty}\left(G\left(\frac{y}{2}\right)-c_{0}\left(\frac{y}{2}\right)\right) y^{s-1} d y \\
\quad+C_{k} \int_{1}^{\infty}\left(H\left(\frac{y}{2}\right)-c_{0}\left(\frac{y}{8}\right)\right) y^{\sigma-k / 2-s-1} d y .
\end{aligned}
$$

We denote the last line in the above by $J_{k}(\sigma, s)$. By Shimura [20, $(2.2)$, Lemma 1], we see that $\tau_{d}(y, \alpha, \beta)$ is holomorphic for $(\alpha, \beta) \in \mathbb{C}^{2}$ and rapidly decreasing for $y \rightarrow \infty$ unless $d=0$. This assures the convergence of the integrals in $J_{k}(\sigma, s)$ as far as the integrands are defined. So seeing the location of poles of the integrands by Proposition 2.2 as a function of $\sigma$, it can be proved that $(\sigma-(k+3) / 2) \zeta(2 \sigma-k-1) J_{k}(\sigma, s)$ is holomorphic at the whole $\sigma, s \in \mathbb{C}$. Hence, $\Phi_{\sigma, k}(s)$ is meromorphically continued to the whole $(\sigma, s) \in \mathbb{C}^{2}$. By Corollary 2.4 , we see easily that $\zeta(2 \sigma-k-1)(\sigma-(k+$ $2) / 2)(\sigma-(k+3) / 2) c(\sigma)$ is holomorphic as a function of $\sigma$, so

$$
\begin{aligned}
& \zeta(2 \sigma-k-1)(\sigma-(k+2) / 2)(\sigma-(k+3) / 2) \\
& \quad \times s(s-1)(s-\sigma+k / 2)(s-\sigma+k / 2+1) \Phi_{\sigma, k}(s)
\end{aligned}
$$

is holomorphic on $(\sigma, s) \in \mathbb{C}^{2}$. In the same way, we see that 


$$
\begin{aligned}
2^{s} \Psi_{\sigma, k}(s)= & \int_{1}^{\infty}\left(H\left(\frac{y}{2}\right)-c_{0}\left(\frac{y}{8}\right)\right) y^{s-1} d y \\
& +C_{k}^{-1} \int_{1}^{\infty}\left(G\left(\frac{y}{2}\right)-c_{0}\left(\frac{y}{2}\right)\right) y^{\sigma-k / 2-s-1} d y \\
& -\int_{1}^{\infty} c_{0}\left(\frac{1}{8 y}\right) y^{-s-1} d y+C_{k}^{-1} \int_{1}^{\infty} c_{0}\left(\frac{y}{2}\right) y^{\sigma-k / 2-s-1} d y \\
= & C_{k}^{-1} J_{k}(\sigma, \sigma-k / 2-s)-\frac{1}{s}-\frac{c(\sigma) 8^{\sigma-k / 2-1}}{s-\sigma+k / 2+1} \\
& -\frac{C_{k}^{-1}}{\sigma-k / 2-s}-\frac{C_{k}^{-1} c(\sigma) 2^{\sigma-k / 2-1}}{1-s} .
\end{aligned}
$$

By comparing this to the expression of $2^{s} \Phi_{\sigma, k}(s)$, we have the following proposition (see [4]).

Proposition 3.2. For a fixed odd $k$, two functions $\Phi_{\sigma, k}(s)$ and $\Psi_{\sigma, k}(s)$ are meromorphically continued to the whole $(\sigma, s) \in \mathbb{C}^{2}$, and we have the following functional equation:

$\Phi_{\sigma, k}(\sigma-k / 2-s)=2^{2 s-\sigma+k / 2} C_{k} \Psi_{\sigma, k}(s)=2^{2 s-2 \sigma+k+1 / 2}(-1)^{\left(k^{2}-1\right) / 8} \Psi_{\sigma, k}(s)$.

\section{2 .}

We will apply functional equations between $\Phi_{\sigma, k}(s)$ and $\Psi_{\sigma, k}(s)$ to obtain those between $D^{*}(s, \delta)$ and $D(s, \delta)$. This can be done by calculating the Mellin transforms term by term and by specializing $\sigma$ and $k$. The Mellin transform of $\tau_{d}(y,(\sigma-k) / 2, \sigma / 2)$ has been given in [4]. For each $\alpha, \beta \in R$ and $s \in C$, we consider the following integral:

$$
I(s, \alpha, \beta)=\int_{0}^{\infty} \frac{(1+u)^{\alpha-1} u^{\beta-1}}{(1+2 u)^{s}} d u .
$$

This integral converges absolutely when $\operatorname{Re}(\beta)>0$ and $\operatorname{Re}(s)>\operatorname{Re}(\alpha)+$ $\operatorname{Re}(\beta)-1$. This is related with the usual hypergeometric functions. This can be shown as follows. For any nonnegative integer $n$ and a variable $a$, we put $(a)_{n}=\Gamma(a+n) / \Gamma(a)$. The Gauss hypergeometric function $F(a, b ; c ; z)$ is by definition

$$
F(a, b ; c ; z)=\sum_{n=0}^{\infty} \frac{(a)_{n}(b)_{n}}{(c)_{n} n !} z^{n}
$$


and has an integral expression

$$
F(a, b ; c ; z)=\frac{\Gamma(c)}{\Gamma(b) \Gamma(c-b)} \int_{0}^{1} t^{b-1}(1-t)^{c-b-1}(1-z t)^{-a} d t
$$

for $\operatorname{Re}(c)>\operatorname{Re}(b)>0$ and $|z|<1$. In the integral defining $I(s, \alpha, \beta)$, by replacing the variable $u$ with $u=v /(2-2 v)$, we have

$$
\begin{aligned}
I(s, \alpha, \beta) & =\frac{1}{2^{\beta}} \int_{0}^{1} v^{\beta-1}(1-v)^{s-\alpha-\beta}\left(1-\frac{v}{2}\right)^{\alpha-1} d v \\
& =\frac{1}{2^{\beta}} \times \frac{\Gamma(\beta) \Gamma(s-\alpha-\beta+1)}{\Gamma(s-\alpha+1)} F(1-\alpha, \beta ; s-\alpha+1 ; 1 / 2) .
\end{aligned}
$$

Now, we assume that $\operatorname{Re}(\sigma)>0, \operatorname{Re}(\sigma)>k$, and $\operatorname{Re}(s)>\operatorname{Re}(\sigma)-k / 2-1$. Then, the Mellin transforms of the Whittaker functions are given by

$$
\begin{aligned}
\int_{0}^{\infty} & \tau_{d}\left(y, \frac{\sigma-k}{2}, \frac{\sigma}{2}\right) y^{s-1} d y \\
= & \frac{e(k / 8)(2 \pi)^{\sigma-k / 2}}{\Gamma((\sigma-k) / 2) \Gamma(\sigma / 2)} \\
& \times \begin{cases}d^{\sigma-k / 2-1}(2 \pi d)^{-s} \Gamma(s) I\left(s, \frac{\sigma-k}{2}, \frac{\sigma}{2}\right) & \text { if } d>0, \\
|d|^{\sigma-k / 2-1}(2 \pi|d|)^{-s} \Gamma(s) I\left(s, \frac{\sigma}{2}, \frac{\sigma-k}{2}\right) & \text { if } d<0 .\end{cases}
\end{aligned}
$$

Hence, if $d=(-1)^{(k+1) / 2} d_{K} f^{2} \neq 0$, we have

$$
\begin{aligned}
\int_{0}^{\infty}{ }_{d}(y) y^{s-1} d y & \\
= & e\left(\frac{k}{8}\right) C\left((-1)^{(k+1) / 2} d_{K} f^{2}, \sigma, k\right)\left(\left|d_{K}\right| f^{2}\right)^{\sigma-k / 2-1-s} \\
& \times \frac{(2 \pi)^{\sigma-k / 2-s} \Gamma(s)}{\Gamma((\sigma-k) / 2) \Gamma(\sigma / 2)} \times \begin{cases}I(s,(\sigma-k) / 2, \sigma / 2) & \text { if } d>0, \\
I(s, \sigma / 2,(\sigma-k) / 2) & \text { if } d<0 .\end{cases}
\end{aligned}
$$

If $\operatorname{Re}(s)>-k / 2-1$, the right-hand side can be continued holomorphically to $\sigma=0$. Indeed, under this condition on $s$ and $k, I(s, \sigma / 2,(\sigma-k) / 2)$ is holomorphic at $\sigma=0$, and we also have

$\frac{1}{\Gamma(\sigma / 2)} I\left(s, \frac{\sigma-k}{2}, \frac{\sigma}{2}\right)=\frac{1}{\Gamma(\sigma / 2)\left(e^{\pi i \sigma}-1\right)} \int_{\infty}^{(0+)} \frac{(u+1)^{(\sigma-k) / 2-1} u^{\sigma / 2-1}}{(2 u+1)^{s}} d u$,

and this function is 1 at $\sigma=0$. 
For the sake of simplicity, we put

$$
\begin{aligned}
& Z^{*}\left(k, \sigma, s, d_{K}\right) \\
& \quad=\left|d_{K}\right|^{\sigma-k / 2-1-s} \frac{L\left(\sigma-(k+1) / 2, \chi_{K}\right) \zeta(2 s) \zeta(2 s-2 \sigma+k+2)}{\zeta(2 \sigma-k-1) L\left(2 s-\sigma+(k+3) / 2, \chi_{K}\right)}
\end{aligned}
$$

and

$$
C_{\sigma, k}=(-1)^{\left(k^{2}-1\right) / 8} \frac{2^{k / 2-\sigma+3 / 2} \pi^{\sigma-k / 2}}{\Gamma((\sigma-k) / 2) \Gamma(\sigma / 2)} .
$$

We write $Z^{*}\left(k, \sigma, s, d_{K}\right)=\sum_{n=1}^{\infty} a(n) n^{-s}$, and we put

$$
Z\left(k, \sigma, s, d_{K}\right)=\sum_{n=1}^{\infty} \frac{a(4 n)}{n^{s}} .
$$

Then, using Proposition 2.2, we have

$$
\begin{aligned}
\Phi_{\sigma, k}(s)= & (2 \pi)^{-s} \Gamma(s) C_{\sigma, k}\left(\sum_{(-1)^{(k+1) / 2} d_{K}>0} Z^{*}\left(k, \sigma, s, d_{K}\right) I\left(s, \frac{\sigma-k}{2}, \frac{\sigma}{2}\right)\right. \\
& \left.+\sum_{(-1)^{(k+1) / 2} d_{K}<0} Z^{*}\left(k, \sigma, s, d_{K}\right) I\left(s, \frac{\sigma}{2}, \frac{\sigma-k}{2}\right)\right)
\end{aligned}
$$

and

$$
\begin{aligned}
\Psi_{\sigma, k}(s)= & (2 \pi)^{-s} \Gamma(s) C_{\sigma, k}\left(\sum_{(-1)^{(k+1) / 2} d_{K}>0} Z\left(k, \sigma, s, d_{K}\right) I\left(s, \frac{\sigma-k}{2}, \frac{\sigma}{2}\right)\right. \\
& \left.+\sum_{(-1)^{(k+1) / 2} d_{K}<0} Z\left(k, \sigma, s, d_{K}\right) I\left(s, \frac{\sigma}{2}, \frac{\sigma-k}{2}\right)\right) .
\end{aligned}
$$

We note that the Dirichlet series $Z^{*}\left(k, \sigma, s, d_{K}\right)$ and $Z\left(k, \sigma, s, d_{K}\right)$ depend on $\sigma-k / 2$ but not on each $\sigma$ and $k$ separately. But the coefficients of these in $\Phi_{\sigma, k}(s)$ or $\Psi_{\sigma, k}(s)$ depend on $k$ and $\sigma$.

Now, in order to give the functional equations we need, we specialize $k$ and $\sigma$ in two different ways.

Case $I$. We put $k=-(n+1)$ and $\sigma=0$. We assume that $n$ is even and that $n \geq 4$. By definition, we have 


$$
\begin{aligned}
& \sum_{(-1)^{n / 2} d_{K}>0} Z^{*}\left(-(n+1), 0, s, d_{K}\right) \\
= & \sum_{(-1)^{n / 2} d_{K}>0}\left|d_{K}\right|^{(n-1) / 2-s} \frac{L\left(n / 2, \chi_{K}\right) \zeta(2 s) \zeta(2 s-n+1)}{\zeta(n) L\left(2 s-n / 2+1, \chi_{K}\right)} \\
= & 2^{-1}(2 \pi)^{n / 2}(-1)^{[n / 4]} \Gamma(n / 2)^{-1} \zeta(n)^{-1} D_{n}^{*}(s, 1) .
\end{aligned}
$$

Hence, we have

$$
\begin{aligned}
\Phi_{0,-n-1}(s)= & (-1)^{n(n+2) / 8+[n / 4]} \pi^{n+1 / 2} \\
& \times \Gamma((n+1) / 2)^{-1} \Gamma(n / 2)^{-1} \zeta(n)^{-1}(2 \pi)^{-s} \Gamma(s) D_{n}^{*}(s, 1) .
\end{aligned}
$$

In the same way, we have

$$
\begin{aligned}
\Psi_{0,-n-1}(s)= & (-1)^{n(n+2) / 8+[n / 4]} \pi^{n+1 / 2} \\
& \times \Gamma((n+1) / 2)^{-1} \Gamma(n / 2)^{-1} \zeta(n)^{-1}(2 \pi)^{-s} \Gamma(s) D_{n}(s, 1) .
\end{aligned}
$$

Hence, by Proposition 3.2, we have

$$
\begin{gathered}
(2 \pi)^{-(n+1) / 2+s} \Gamma\left(\frac{n+1}{2}-s\right) D_{n}^{*}\left(\frac{n+1}{2}-s, 1\right) \\
=2^{2 s-(n+1) / 2}(2 \pi)^{-s} \Gamma(s) D_{n}(s, 1) C_{k},
\end{gathered}
$$

where $C_{k}=2^{-n / 2}(-1)^{n(n+2) / 8}$. Since

$$
\Gamma((n+1) / 2-s)^{-1}=(-1)^{n / 2} \pi^{-1}(\cos \pi s) \Gamma(s-(n-1) / 2),
$$

we have the following proposition.

Proposition 3.3. For any even positive integer $n \geq 4$, we have

$$
\begin{aligned}
D_{n}^{*}\left(\frac{n+1}{2}-s, 1\right)= & (-1)^{n(n-2) / 8} 2^{-n / 2} \pi^{-2 s+(n-1) / 2} \\
& \times \Gamma(s) \Gamma\left(s-\frac{n-1}{2}\right)(\cos \pi s) D_{n}(s, 1) .
\end{aligned}
$$

When $n=2$ and $\delta=-1$, we need some modification of the definition of the zeta function and the functional equation, since $L\left(1, \chi_{K}\right)=\infty$ if $K=\mathbb{Q} \oplus \mathbb{Q}$. But if $\delta=1$, the definitions of $D_{2}(s, 1)$ and $D_{2}^{*}(s, 1)$ are the same as in the case $n \geq 4$, and we have the following result. 
Proposition 3.4. We have

$$
D_{2}^{*}(3 / 2-s, 1)=2^{-1} \pi^{1 / 2-2 s} \Gamma(s) \Gamma(s-1 / 2)\left(\cos (\pi s) D_{2}(s, 1)-\zeta(2 s-1)\right) .
$$

The Dirichlet series $D_{2}^{*}(s, 1)$ and $D_{2}(s, 1)$ are continued meromorphically to the whole $s$ plane and are holomorphic for $s \neq 1, s \neq 3 / 2$. The residue is $-1 / 2$ or $-1 / 4$ at $s=1$ for $D_{2}(s, 1)$ or $D_{2}^{*}(s, 1)$, respectively, and $\pi / 3$ or $\pi / 12$ at $s=3 / 2$, respectively.

This proposition was first proved by Siegel [22] and Shintani [21] without using Eisenstein series. (Incidentally, in Shintani's notation, $D_{2}(s, 1)=$ $2^{2 s} \xi_{-}^{*}(s)$ and $D_{2}^{*}(s, 1)=\xi_{-}(s)$.) Later, an alternative proof in the case $n=2$ is obtained by Sturm [23]. His argument is to use the Eisenstein series above, noting that, in the above calculation, $I(s, 0,3 / 2)$ is holomorphic for $s>1 / 2$ and that $\zeta(\sigma+1) / \Gamma(\sigma / 2)$ is holomorphic at $\sigma=0$. For the case $(n, \delta)=(2,-1)$, we need a modification of the definition, which will be explained in Section 5.

Case II. We put $k=-n+3$ and $\sigma=2$ with even $n \geq 4$. By changing the variable $u$ by $v=2 u(2 u+1)^{-1}$, we have

$$
\begin{aligned}
I\left(s, 1, \frac{n-1}{2}\right) & =2^{-(n-1) / 2} \int_{0}^{1} v^{(n-3) / 2}(1-v)^{s-(n+1) / 2} d v \\
& =2^{-(n-1) / 2} \Gamma(s)^{-1} \Gamma\left(\frac{n-1}{2}\right) \Gamma\left(s-\frac{n-1}{2}\right) .
\end{aligned}
$$

On the other hand, the function $I(s,(n-1) / 2,1)$ can be continued meromorphically to the whole $s$ plane and satisfies

$$
\begin{aligned}
& I\left(s, \frac{n-1}{2}, 1\right)+I\left(\frac{n+1}{2}-s, \frac{n-1}{2}, 1\right) \\
& \quad=2^{-(n-1) / 2} \Gamma\left(\frac{3-n}{2}\right)^{-1} \Gamma(1-s) \Gamma\left(s-\frac{n-1}{2}\right) .
\end{aligned}
$$

This can be proved easily by using the Pochhammer integral formula for the beta function. Indeed, put $v=(1+2 u) /(2+2 u)$. Then,

$$
I\left(s, \frac{n-1}{2}, 1\right)=2^{-(n-1) / 2} \int_{1 / 2}^{1} v^{-s}(1-v)^{s-(n+1) / 2} d v
$$

Now, for $\alpha, \beta>0$, put $f(x)=x^{\alpha-1}(1-x)^{\beta-1}$. Take a positive branch at $x=1 / 2$, and take an analytic continuation along the path starting at $1 / 2$, 
encircling 1 and 0 in the positive direction and then encircling 1 and 0 in the negative direction. Then,

$$
\int_{1 / 2}^{(1+, 0+, 1-, 0-)} x^{\alpha}(1-x)^{\beta} d x=\frac{1}{\left(1-e^{2 \pi i \alpha}\right)\left(1-e^{2 \pi i \beta}\right)} B(\alpha, \beta),
$$

where $B(\alpha, \beta)=\Gamma(\alpha) \Gamma(\beta) \Gamma(\alpha+\beta)^{-1}$ is the usual beta function. We also have

$$
\int_{1 / 2}^{1} v^{\alpha}(1-v)^{\beta} d v=\frac{1}{\left(1-e^{2 \pi i \alpha}\right)\left(1-e^{2 \pi i \beta}\right)}\left(\int_{1 / 2}^{(1+)} f(x) d x+\int_{1 / 2}^{(1-)} f(x) d x\right)
$$

and

$$
\int_{0}^{1 / 2} v^{\alpha}(1-v)^{\beta} d v=\frac{1}{\left(1-e^{2 \pi i \alpha}\right)\left(1-e^{2 \pi i \beta}\right)}\left(\int_{1 / 2}^{(0+)} f(x) d x+\int_{1 / 2}^{(0-)} f(x) d x\right),
$$

and we obtain the above functional equation of $I(s,(n-1) / 2,1)$. By virtue of the integral expressions above, $\Gamma(s-(n-1) / 2)^{-1} I(s,(n-1) / 2,1)$ is an entire function, and we see that $I(s,(n-1) / 2,1)$ has simple poles only at $s=(n+1) / 2-m(m=1,2, \ldots)$. The residue for each $m$ is given by $(-1)^{m-1} 2^{-(n-1) / 2} \Gamma(m-(n-1) / 2) \Gamma((3-n) / 2)^{-1} \Gamma(m)^{-1}$.

By definition, we have

$$
\begin{aligned}
\Phi_{2,-n+3}(s)= & (-1)^{n(n+2) / 8+1+[n / 4]} \pi^{n+1 / 2} \\
& \times \Gamma(n / 2)^{-1} \Gamma((n-1) / 2)^{-1} \zeta(n)^{-1}(2 \pi)^{-s} \Gamma(s) \\
& \times\left(D_{n}^{*}(s, 1) I\left(s, \frac{n-1}{2}, 1\right)+D_{n}^{*}(s,-1) I\left(s, 1, \frac{n-1}{2}\right)\right)
\end{aligned}
$$

and

$$
\begin{aligned}
\Psi_{2,-n+3}(s)= & (-1)^{n(n+2) / 8+1+[n / 4]} \pi^{n+1 / 2} \\
& \times \Gamma(n / 2)^{-1} \Gamma((n-1) / 2)^{-1} \zeta(n)^{-1}(2 \pi)^{-s} \Gamma(s) \\
& \times\left(D_{n}(s, 1) I\left(s, \frac{n-1}{2}, 1\right)+D_{n}(s,-1) I\left(s, 1, \frac{n-1}{2}\right)\right) .
\end{aligned}
$$

Hence, by Proposition 3.2, we have 


$$
\begin{aligned}
(2 \pi)^{s-} & (n+1) / 2 \Gamma\left(\frac{n+1}{2}\right) \\
& \times\left(D_{n}^{*}\left(\frac{n+1}{2}-s, 1\right) I\left(\frac{n+1}{2}-s, \frac{n-1}{2}, 1\right)\right. \\
& \left.+D_{n}^{*}\left(\frac{n+1}{2}-s,-1\right) I\left(\frac{n+1}{2}-s, 1, \frac{n-1}{2}\right)\right) \\
= & 2^{2 s-n-1 / 2}(-1)^{n(n+2) / 8+1}(2 \pi)^{-s} \Gamma(s) \\
& \times\left(D_{n}(s, 1) I\left(s, \frac{n-1}{2}, 1\right)+D_{n}(s,-1) I\left(s, 1, \frac{n-1}{2}\right)\right) .
\end{aligned}
$$

Here, using the functional equation between $D_{n}^{*}(s, 1)$ and $D_{n}(s, 1)$ and the formulas for $I(s, 1,(n-1) / 2)$ and $I(s,(n-1) / 2,1)$, we have

$$
\begin{aligned}
(2 \pi)^{s-} & (n+1) / 22^{-(n-1) / 2} \Gamma\left(\frac{n-1}{2}\right) \Gamma(1-s) D_{n}^{*}\left(\frac{n+1}{2}-s,-1\right) \\
= & 2^{2 s-n-1 / 2}(-1)^{n(n+2) / 8+1}(2 \pi)^{-s} \Gamma(s) 2^{-(n-1) / 2} \\
& \times\left(\Gamma\left(\frac{3-n}{2}\right)^{-1} D_{n}(s, 1) \Gamma(1-s) \Gamma\left(s-\frac{n-1}{2}\right)\right. \\
& \left.+\Gamma\left(\frac{n-1}{2}\right) D_{n}(s,-1) \Gamma(s)^{-1} \Gamma\left(s-\frac{n-1}{2}\right)\right) .
\end{aligned}
$$

Since $\Gamma(s) \Gamma(1-s)=\pi / \sin \pi s$ and $\Gamma((n-1) / 2) \Gamma((3-n) / 2)=\pi / \sin (((n-$ 1) $/ 2) \pi)=\pi(-1)^{n / 2+1}$, we have the following proposition.

Proposition 3.5. For even $n \geq 4$, we have

$$
\begin{aligned}
D_{n}^{*}\left(\frac{n+1}{2}-s,-1\right)= & 2^{-n / 2} \pi^{-2 s+(n-1) / 2}(-1)^{n(n+2) / 8+1} \Gamma(s) \Gamma\left(s-\frac{n-1}{2}\right) \\
& \times\left((\sin \pi s) D_{n}(s,-1)+(-1)^{n / 2+1} D_{n}(s, 1)\right) .
\end{aligned}
$$

For the necessary modification in case $n=2$, see Section 5 .

For later use, we will give poles and residues of $D_{n}^{*}(s, \delta)$ and $D_{n}(s, \delta)$ $(\delta= \pm 1)$. If $\sigma=0$ and $k=-(n+1)$, then $c_{0}(y)=1$. So $\Phi_{0, k}(s)$ or $\Psi_{0, k}(s)$ has simple poles only at $s=0$ and $(n+1) / 2$.

Proposition 3.6. The Dirichlet series $D_{n}^{*}(s, 1)$ or $D_{n}(s, 1)$ is holomorphic except for a simple pole at $s=(n+1) / 2$ with the residue $(-1)^{[n / 4]} \times$ $(2 \pi)^{-n / 2} \Gamma(n / 2) \zeta(n)$ or $(-1)^{[n / 4]} 2^{n / 2} \pi^{-n / 2} \Gamma(n / 2) \zeta(n)$, respectively. The 
Dirichlet series $D_{n}^{*}(s,-1)$ or $D_{n}(s,-1)$ is holomorphic except for simple poles at $s=1$ and $s=(n+1) / 2$. The residue at $s=1$ is $\pi^{-n-1 / 2} \Gamma(n / 2) \times$ $\Gamma((n-1) / 2) \zeta(n-1)$ or $2 \pi^{-n-1 / 2} \Gamma(n / 2) \Gamma((n-1) / 2) \zeta(n-1)$, and the residue at $s=(n+1) / 2$ is $(-1)^{[n / 4]}(2 \pi)^{-n / 2} \Gamma(n / 2) \zeta(n)$ or $(-1)^{[n / 4]} 2^{n / 2} \pi^{-n / 2} \Gamma(n /$ 2) $\zeta(n)$, respectively.

Proof. Since $\Phi_{0,-n-1}(s)$ or $\Psi_{0,-n-1}(s)$ has a simple pole at $s=0$, $\Phi_{0,-n-1}(s) / \Gamma(s)$ or $\Psi_{0,-n-1}(s) / \Gamma(s)$ is holomorphic at $s=0$. Hence, $D_{n}^{*}(s, 1)$ or $D_{n}(s, 1)$ is holomorphic for $s \neq(n+1) / 2$. At $s=(n+1) / 2$, the residue of $\Phi_{0,-n-1}(s)$ or $\Psi_{0,-n-1}(s)$ is $2^{-n-1 / 2}(-1)^{n(n+2) / 8}$ or $2^{-1 / 2}(-1)^{n(n+2) / 8}$. Hence, we have our results for $D_{n}^{*}(s, \delta)$ and $D_{n}(s, \delta)$ for $\delta=1$. Next, we treat the case $\delta=-1$. By the results in Section 3.1, for even $n \geq 4$, we see that $\Phi_{2,-n+3}(s)$ and $\Psi_{2,-n+3}(s)$ are holomorphic except for $s=0,1$, $(n-1) / 2$, and $(n+1) / 2$ and that $\Gamma(s) D_{n}^{*}(s, 1)$ or $\Gamma(s) D_{n}(s, 1)$ has simple poles only at $s=0,(n+1) / 2$. Since $\Gamma(s-(n-1)) / 2)^{-1} I(s,(n-1) / 2,1)$ is entire, it is easy to see that $D_{n}^{*}(s,-1)$ and $D_{n}(s,-1)$ are holomorphic at $s \neq 0,1$, and $(n+1) / 2$. Now, by Proposition 3.3 and the above result, we have

$$
D_{n}^{*}(0,1)=D_{n}(0,1)=(-1)^{n(n+2) / 8+1+[n / 4]} \pi^{-n-1 / 2} \Gamma(n / 2) \Gamma((n+1) / 2) \zeta(n) .
$$

Since the residue of $\Phi_{2,-n+3}(s)$ and $\Psi_{2,-n+3}(s)$ at $s=0$ is -1 and $I(0,(n-$ $1) / 2,1)=-2 /(n-1)$, we see that $D_{n}^{*}(s,-1)$ and $D_{n}(s,-1)$ are holomorphic at $s=0$. The residue of $\Phi_{2,-n+3}(s)$ or $\Psi_{2,-n+3}(s)$ at $s=(n+1) / 2$ is $2^{-n-1 / 2}(-1)^{n(n+2) / 8+1}$ or $2^{-1 / 2}(-1)^{n(n+2) / 8+1}$ and at $s=1$ is $2^{-(n+1) / 2} \times$ $\zeta(n-1) \zeta(n)^{-1}$ or $2^{(1-n) / 2} \zeta(n-1) \zeta(n)^{-1}$, respectively. Using $I((n+1) / 2$, $(n-1) / 2,1)=2 \times\left(1-2^{(1-n) / 2}\right) /(n-1)$ and the estimates of residues of $D_{n}(s, 1)$ and $D_{n}^{*}(s, 1)$ at $s=(n+1) / 2$ just obtained, we can easily obtain the residues for $\delta=-1$.

\subsection{Functional equation of Dirichlet series of two variables}

In [21] Shintani introduced certain Dirichlet series of two variables $\xi_{i}\left(s_{1}, s_{2}\right)$ and $\xi_{i}^{*}\left(s_{1}, s_{2}\right)$ and used the functional equation between them to derive a similar result on Dirichlet series of one variable such as those in Section 3.2 (see [21]). In the review of this paper, the referee asked if we can prove his functional equation of two variables by our method. Indeed, we can prove it, and we sketch the proof here. For a positive integer $m$ and an integer $n$, we write $A(m, n)$ the number of $x \bmod m$ such that $x^{2} \equiv n \bmod m$. 
For $i=1,2$, following Shintani [21], we define

$$
\begin{aligned}
& \xi_{i}\left(s_{1}, s_{2}\right)=\sum_{m, n=1}^{\infty} A\left(4 m,(-1)^{i-1} n\right) m^{-s_{1}} n^{-s_{2}}, \\
& \xi_{i}^{*}\left(s_{1}, s_{2}\right)=\sum_{m, n=1}^{\infty} A\left(m,(-1)^{i-1}\right) m^{-s_{1}}(4 n)^{-s_{2}} .
\end{aligned}
$$

(Note that in [21] there is a typo in the introduction and that the definition at $[21$, p. 35$]$ is the correct one.) We also put

$$
\begin{aligned}
& \eta_{i}^{*}(\sigma, s)=\sum_{(-1)^{i-1} d_{K}>0} Z^{*}\left(k, \sigma+\frac{k+1}{2}, s, d_{K}\right), \\
& \eta_{i}(\sigma, s)=\sum_{(-1)^{i-1} d_{K}>0} Z\left(k, \sigma+\frac{k+1}{2}, s, d_{K}\right) .
\end{aligned}
$$

We note that these series do not depend on $k$, while the coefficients in $\Phi_{\sigma+(k+1) / 2, k}$ and $\Psi_{\sigma+(k+1) / 2, k}$ do so. By applying [27], we see easily that

$$
\begin{aligned}
& \eta_{i}^{*}(\sigma, s)=\zeta(\sigma)^{-1} \xi_{i}\left(\sigma, s+\frac{1}{2}-\sigma\right) \\
& \eta_{i}(\sigma, s)=2^{2 s} \zeta(\sigma)^{-1} \xi_{i}^{*}\left(\sigma, s+\frac{1}{2}-\sigma\right) .
\end{aligned}
$$

Our aim here is to reprove the functional equation in [21, Theorem 1], which is equivalent to the following.

Proposition 3.7. We have

$$
\left(\begin{array}{l}
\eta_{1}^{*}\left(\sigma, \sigma+\frac{1}{2}-s\right) \\
\eta_{2}^{*}\left(\sigma, \sigma+\frac{1}{2}-s\right)
\end{array}\right)=\frac{\Gamma(s) \Gamma\left(s+\frac{1}{2}-\sigma\right)}{2^{\sigma} \pi^{2 s-\sigma+1 / 2}} M(s)\left(\begin{array}{l}
\eta_{1}(\sigma, s) \\
\eta_{2}(\sigma, s)
\end{array}\right)
$$

where we put

$$
M(s)=\left(\begin{array}{cc}
\cos \pi\left(s-\frac{\sigma}{2}\right) & \sin \frac{\pi \sigma}{2} \\
\cos \frac{\pi \sigma}{2} & -\sin \pi\left(s-\frac{\sigma}{2}\right)
\end{array}\right) .
$$

By virtue of Proposition 3.2, we can obtain the functional equation between $\eta_{i}^{*}$ and $\eta_{i}$ as far as we have relations between $I(*, \alpha, \beta)$ and $I(*, \alpha, \beta)$ for $(\alpha, \beta)=((\sigma / 2)+(-k+1) / 4,(\sigma / 2)+(k+1) / 4)$ which appear in 
$\Phi_{\sigma+(k+1) / 2, k}$ or $\Psi_{\sigma+(k+1) / 2, k}$. Now such relations are obtained as follows. The following relations of the hypergeometric functions are well known:

$$
\begin{aligned}
F(a, b ; c: z)= & \frac{\Gamma(c) \Gamma(c-a-b)}{\Gamma(c-a) \Gamma(c-b)} F(a, b: a+b-c+1,1-z) \\
& +(1-z)^{c-a-b} \frac{\Gamma(c) \Gamma(a+b-c)}{\Gamma(a) \Gamma(b)} \\
& \times F(c-a, c-b ; c-a-b+1 ; 1-z)
\end{aligned}
$$

and

$$
F(a, b ; c ; z)=(1-z)^{c-a-b} F(c-a, c-b ; c ; z) .
$$

By taking $z=1 / 2$ in the above relations, we have

$$
\begin{aligned}
I(s, \alpha, \beta)= & \frac{\Gamma(\beta) \Gamma(s-\beta)}{2^{\beta} \Gamma(s)} F(1-\alpha, \beta ; \beta-s+1 ; 1 / 2) \\
& +\frac{1}{2^{\alpha}} \frac{\Gamma(s-\alpha-\beta+1) \Gamma(\beta-s)}{\Gamma(1-\alpha)} F(1-\beta, \alpha ; s-\beta+1 ; 1 / 2) .
\end{aligned}
$$

Here the first term is equal to

$$
\frac{\Gamma(s-\beta) \Gamma(\beta-s+1)}{\Gamma(s) \Gamma(1-s)} I(\alpha+\beta-s, \alpha, \beta),
$$

and the second term is equal to

$$
\frac{\Gamma(\beta-s) \Gamma(s-\beta+1)}{\Gamma(1-\alpha) \Gamma(\alpha)} I(s, \beta, \alpha) .
$$

Hence, we have

$$
\begin{aligned}
I(s, \alpha, \beta)= & \frac{\Gamma(s-\beta) \Gamma(\beta-s+1)}{\Gamma(s) \Gamma(1-s)} I(\alpha+\beta-s, \alpha, \beta) \\
& +\frac{\Gamma(\beta-s) \Gamma(s-\beta+1)}{\Gamma(1-\alpha) \Gamma(\alpha)} I(s, \beta, \alpha) .
\end{aligned}
$$

Since $\Gamma(s-\beta) \Gamma(\beta-s+1)=\pi /(\sin \pi(s-\beta))=-\Gamma(\beta-s) \Gamma(s-\beta+1)$, we have

$$
I(\alpha+\beta-s)=\frac{\Gamma(s) \Gamma(1-s)}{\Gamma(s-\beta) \Gamma(\beta-s+1)} I(s, \alpha, \beta)+\frac{\Gamma(s) \Gamma(1-s)}{\Gamma(1-\alpha) \Gamma(\alpha)} I(s, \beta, \alpha),
$$




$$
I(\alpha+\beta-s)=\frac{\Gamma(s) \Gamma(1-s)}{\Gamma(s-\alpha) \Gamma(\alpha-s+1)} I(s, \beta, \alpha)+\frac{\Gamma(s) \Gamma(1-s)}{\Gamma(1-\beta) \Gamma(\beta)} I(s, \alpha, \beta) .
$$

In other words, if we put

$$
N(s, \alpha, \beta)=\left(\begin{array}{cc}
\sin \pi(s-\beta) & \sin \pi \beta \\
\sin \pi \alpha & \sin \pi(s-\alpha)
\end{array}\right),
$$

then we have

$$
\begin{aligned}
& (I(\alpha+\beta-s, \alpha, \beta), I(\alpha+\beta-s, \beta, \alpha)) \\
& \quad=(I(s, \alpha, \beta), I(s, \beta, \alpha)) \times N(s, \alpha, \beta) \times \frac{1}{\sin \pi s} .
\end{aligned}
$$

By taking $k \equiv 7 \bmod 8$ and $\alpha \equiv(\sigma / 2)+(1 / 2) \bmod 2, \beta \equiv \sigma / 2 \bmod 2$, we have

$$
N(s, \alpha, \beta)=\left(\begin{array}{cc}
\sin \pi\left(s-\frac{\sigma}{2}\right) & \sin \frac{\pi \sigma}{2} \\
\cos \frac{\pi \sigma}{2} & -\cos \pi\left(s-\frac{\sigma}{2}\right)
\end{array}\right) .
$$

By noting that

$$
\sin \pi s \sin \pi\left(s+\frac{1}{2}-\sigma\right)=\sin \frac{\pi \sigma}{2} \cos \frac{\pi \sigma}{2}+\sin \pi\left(s-\frac{\sigma}{2}\right) \cos \pi\left(s-\frac{\sigma}{2}\right),
$$

we have

$$
\sin \pi s \sin \pi\left(s+\frac{1}{2}-\sigma\right) N(s, \alpha, \beta)^{-1}=M(s) .
$$

We can do the same calculation for each odd $k \bmod 8$. Denote by $v(k)$ a 2 dimensional vector whose components are coefficients (including $C_{\sigma+(k+1) / 2, k}$ ) of $\eta_{1}^{*}(\sigma, s)$ and $\eta_{2}^{*}(\sigma, s)$ in $\Phi_{\sigma+(k+1) / 2}(s)$. Then for $k_{1} \equiv 7 \bmod 8$ and $k_{2} \equiv$ $3 \bmod 8$, two vectors $v\left(k_{1}\right)$ and $v\left(k_{2}\right)$ are linearly independent over meromorphic functions as is shown in the argument in Section 3.2. Then by using the functional equation in Proposition 3.2 and the above formula for $N(s, \alpha, \beta)$, we can easily prove Proposition 3.7 by direct calculation. Propositions 3.3 and 3.5 can be seen as special cases of Proposition 3.7. We omit the details here.

\section{§4. Proof of functional equations for $n \geq 3$}

The functional equations for the case where $n \geq 3$, as well as the residues, are easily obtained from the well-known facts on the Riemann zeta function 
and results on $D^{*}(s, \delta)$ and $D(s, \delta)$ in Section 3 . We carry out this calculation in this section. By using the well-known functional equation

$$
\begin{aligned}
\zeta(1-s) & =\pi^{-s-1 / 2}\left(\cos \frac{\pi s}{2}\right) \Gamma\left(\frac{s}{2}\right) \Gamma\left(\frac{s+1}{2}\right) \zeta(s) \\
& =\pi^{-s} 2^{1-s}\left(\cos \frac{\pi s}{2}\right) \Gamma(s) \zeta(s),
\end{aligned}
$$

we have

$$
\begin{aligned}
& \zeta\left(2\left(\frac{n+1}{2}-s\right)-(2 i-1)\right) \\
& =\pi^{-2 s+n-2 i+1 / 2} \Gamma\left(s+i-\frac{n}{2}\right) \Gamma\left(s+i-\frac{n+1}{2}\right) \zeta(2 s+2 i-n-1) \\
& \quad \times \begin{cases}(-1)^{i-(n+1) / 2} \cos \pi s & \text { if } n \text { is odd, } \\
(-1)^{i-n / 2} \sin \pi s & \text { if } n \text { is even, }\end{cases} \\
& \zeta\left(2\left(\frac{n+1}{2}-s\right)-2 i\right) \\
& =\pi^{-2 s+n-2 i-1 / 2} \Gamma\left(s+i-\frac{n}{2}\right) \Gamma\left(s+i-\frac{n-1}{2}\right) \zeta(2 s+2 i-n) \\
& \quad \times \begin{cases}(-1)^{i-(n-1) / 2} \sin \pi s & \text { if } n \text { is odd, } \\
(-1)^{i-n / 2} \cos \pi s & \text { if } n \text { is even. }\end{cases}
\end{aligned}
$$

Hence, for even $n \geq 4$, we have

$$
\begin{aligned}
A_{n}\left(\frac{n+1}{2}-s\right)= & (-1)^{n(n-2) / 8} \pi^{-(n-2) s+n(n-3) / 4+1 / 2} \\
& \times(\cos \pi s)^{\frac{n}{2}-1} \frac{\gamma_{n}\left(s-\frac{n+1}{2}\right)}{\Gamma(s) \Gamma\left(s-\frac{n-1}{2}\right)} A_{n}(s), \\
B_{n}\left(\frac{n+1}{2}-s\right)= & (-1)^{n(n-2) / 8} \pi^{-n s+n(n-1) / 4} \gamma_{n}\left(s-\frac{n+1}{2}\right)(\sin \pi s)^{n / 2} B_{n}(s) .
\end{aligned}
$$

Using Propositions 3.3 and 3.5, we have

$$
\begin{aligned}
& D_{n}^{*}\left(\frac{n+1}{2}-s, 1\right) A_{n}\left(\frac{n+1}{2}-s\right) \\
& \quad=2^{-n / 2} \pi^{-n s+n(n-1) / 4}(\cos \pi s)^{n / 2} \gamma_{n}\left(s-\frac{n+1}{2}\right) A_{n}(s) D_{n}(s, 1) \\
& D_{n}^{*}\left(\frac{n+1}{2}-s,-1\right) A_{n}\left(\frac{n+1}{2}-s\right)
\end{aligned}
$$




$$
\begin{aligned}
= & 2^{-n / 2} \pi^{-n s+n(n-1) / 4} \gamma_{n}\left(s-\frac{n+1}{2}\right) A_{n}(s) \\
& \times\left((-1)^{n^{2} / 4+1}(\cos \pi s)^{n / 2-1}(\sin \pi s) D_{n}(s,-1)\right. \\
& \left.+(\cos \pi s)^{n / 2-1} D_{n}(s, 1)\right) .
\end{aligned}
$$

For odd $n \geq 3$, we have

$$
\begin{aligned}
Q_{n}\left(\frac{n+1}{2}-s\right)= & \pi^{-n s+n(n-1) / 4} 2^{1-s}(-1)^{\left(n^{2}-1\right) / 8} \\
& \times \gamma_{n}\left(s-\frac{n+1}{2}\right)\left(\cos \frac{\pi s}{2}\right)(\cos \pi s)^{(n-1) / 2} R_{n}(s), \\
R_{n}\left(\frac{n+1}{2}-s\right)= & \pi^{-n s+n(n-1) / 4} 2^{(n+1) / 2-s} \\
& \times \gamma_{n}\left(s-\frac{n+1}{2}\right)(\sin \pi s)^{(n-1) / 2} Q_{n}(s) \\
& \times \begin{cases}\cos \frac{\pi s}{2} & \text { if } n \equiv 1 \bmod 4, \\
\sin \frac{\pi s}{2} & \text { if } n \equiv 3 \bmod 4 .\end{cases}
\end{aligned}
$$

Now, the functional equations in Section 1 are proved as follows. We put $\epsilon_{0}=\epsilon \delta^{(n+1) / 2}$.

When $n$ is odd, then for $L=L_{n}^{*}$ or $L_{n}$, the zeta function $\zeta(s, L, \epsilon, \delta)$ depends only on $\epsilon_{0}$. For the sake of simplicity, we put $\zeta(s, L, \epsilon, \delta)=\zeta^{+}(s, L)$ or $\zeta^{-}(s, L)$, if $\epsilon=1$ or -1 , respectively. We have

$$
\begin{aligned}
\zeta( & \left.\frac{n+1}{2}-s, L_{n}^{*}, \delta, \epsilon\right) \\
= & b_{n} 2^{(n-1)((n+1) / 2-s)}\left(Q_{n}\left(\frac{n+1}{2}-s\right)+\epsilon_{0}(-1)^{\left(n^{2}-1\right) / 8} R_{n}\left(\frac{n+1}{2}-s\right)\right) \\
= & \pi^{-n s+n(n-1) / 4} 2^{-n s+\left(n^{2}+1\right) / 2} \gamma\left(s-\frac{n+1}{2}\right) \\
& \times\left(\cos \frac{\pi s}{2}(\cos \pi s)^{(n-1) / 2} R_{n}(s)\right. \\
& \left.\quad+(-1)^{\left(n^{2}-1\right) / 8} 2^{(n-1) / 2} \epsilon_{0}(\sin \pi s)^{(n-1) / 2} f(s) Q_{n}(s)\right)
\end{aligned}
$$

where we denote $f(s)=\cos (\pi s / 2)$ or $\sin (\pi s / 2)$, if $n \equiv 1 \bmod 4$ or $3 \bmod 4$, respectively. We have the following equalities easily from the definitions:

$$
Q_{n}(s)=b_{n}^{-1} 2^{-(n+1) / 2}\left(\zeta^{+}\left(s, L_{n}\right)+\zeta^{-}\left(s, L_{n}\right)\right),
$$




$$
R_{n}(s)=b_{n}^{-1} 2^{-1}\left(\zeta^{+}\left(s, L_{n}\right)-\zeta^{-}\left(s, L_{n}\right)\right)
$$

Hence, we have

$$
\begin{aligned}
\zeta^{ \pm} & \left(\frac{n+1}{2}-s, L_{n}^{*}\right) \\
= & (2 \pi)^{-n s} \pi^{n(n-1) / 4} 2^{\left(n^{2}-1\right) / 2} \gamma_{n}\left(s-\frac{n+1}{2}\right) \\
& \quad \times\left(\left(\cos \frac{\pi s}{2}(\cos \pi s)^{(n-1) / 2} \pm(-1)^{\left(n^{2}-1\right) / 8}(\sin \pi s)^{(n-1) / 2} f(s)\right) \zeta^{+}\left(s, L_{n}\right)\right. \\
& +\left(-\cos \frac{\pi s}{2}(\cos \pi s)^{(n-1) / 2} \pm(-1)^{\left(n^{2}-1\right) / 8}(\sin \pi s)^{(n-1) / 2} f(s)\right) \\
& \left.\times \zeta^{-}\left(s, L_{n}\right)\right)
\end{aligned}
$$

Thus, we have proved Theorem 1.

Next, we treat the case when $n$ is even. We prove the functional equation for each $\delta$ and $n$.

Case $I$. Let $\delta=1$, and let $n \equiv 0 \bmod 4$. Then, $\delta_{n}=1$, and

$$
\begin{aligned}
b_{n}(-1)^{[n / 4]} D_{n}(s, 1) A_{n}(s) & =2^{-1}\left(\zeta\left(s, L_{n}, 1,1\right)+\zeta\left(s, L_{n}, 1,-1\right)\right), \\
b_{n}(-1)^{n(n+2) / 8} \frac{2\left|B_{n / 2}^{\prime}\right|}{n} B_{n}(s) & =2^{-n / 2-1}\left(\zeta\left(s, L_{n}, 1,1\right)-\zeta\left(s, L_{n}, 1,-1\right)\right) .
\end{aligned}
$$

So, we have

$$
\begin{aligned}
& \zeta\left(\frac{n+1}{2}-s, L_{n}^{*}, 1, \epsilon\right) \\
& =2^{-n s+n^{2} / 2-1} \pi^{-n s+n(n-1) / 4} \gamma_{n}\left(s-\frac{n+1}{2}\right) \\
& \quad \times\left((\cos \pi s)^{n / 2} \times\left(\zeta\left(s, L_{n}, 1,1\right)+\zeta\left(s, L_{n}, 1,-1\right)\right)\right. \\
& \left.\quad+\epsilon(-1)^{n(n-2) / 8}(\sin \pi s)^{n / 2} \times\left(\zeta\left(s, L_{n}, 1,1\right)-\zeta\left(s, L_{n}, 1,-1\right)\right)\right) .
\end{aligned}
$$

Case II. Let $\delta=-1$, and let $n \equiv 0 \bmod 4$. Then $\delta_{n}=0$, and the zeta functions do not depend on $\epsilon$ :

$$
\begin{aligned}
& \zeta\left(\frac{n+1}{2}-s, L_{n}^{*},-1, \epsilon\right) \\
& \quad=2^{-n s-n^{2} / 2} \pi^{-n s+n(n-1) / 4} \gamma_{n}\left(s-\frac{n+1}{2}\right)
\end{aligned}
$$




$$
\begin{aligned}
& \times\left((-1)^{n^{2} / 4+1}(\cos \pi s)^{n / 2-1}(\sin \pi s) \zeta\left(s, L_{n},-1, \epsilon\right)\right. \\
& \left.+(\cos \pi s)^{n / 2-1} 2^{-1}\left(\zeta\left(s, L_{n}, 1,1\right)-\zeta\left(s, L_{n}, 1,-1\right)\right)\right)
\end{aligned}
$$

Case III. Let $\delta=1$, and let $n \equiv 2 \bmod 4$ with $n \geq 4$. Then, the zeta functions do not depend on $\epsilon$. In this case, we have

$$
\begin{aligned}
\zeta\left(\frac{n+1}{2}-s, L_{n}^{*}, 1, \epsilon\right)= & 2^{n(n+1 / 2-s)} 2^{-n / 2} \pi^{-n s+n(n-1) / 4} \\
& \times(\cos \pi s)^{n / 2} \gamma_{n}\left(s-\frac{n+1}{2}\right) \zeta\left(s, L_{n}, 1, \epsilon\right) .
\end{aligned}
$$

Case $I V$. Let $\delta=-1$, and let $n \equiv 2 \bmod 4$ with $n \geq 4$. In this case, $\delta_{n}=1$ and

$$
\begin{aligned}
\zeta( & \left.\frac{n+1}{2}-s, L_{n}^{*},-1, \epsilon\right) \\
= & 2^{-n s-n^{2} / 2} \pi^{-n s+n(n-1) / 4} \gamma_{n}\left(s-\frac{n+1}{2}\right) \\
& \quad \times\left((-1)^{n^{2} / 4+1} 2^{-1}(\cos \pi s)^{n / 2-1}(\sin \pi s)\right. \\
& \quad \times\left(\zeta\left(s, L_{n},-1,1\right)+\zeta\left(s, L_{n},-1,-1\right)\right) \\
& +(-1)^{n(n-2) / 8} 2^{-1} \epsilon(\sin \pi s)^{n / 2}\left(\zeta\left(s, L_{n},-1,1\right)-\zeta\left(s, L_{n},-1,-1\right)\right) \\
& \left.+(\cos \pi s)^{n / 2-1} \zeta\left(s, L_{n}, 1, \epsilon\right)\right) .
\end{aligned}
$$

Hence, we have proved Theorem 3 .

As is well known, $\zeta(s)$ has a simple pole at $s=1$ with residue 1 and is holomorphic for the other $s$. Also, we have $\zeta(-2 m)=0$ for all positive integers $m$. Theorem 2 follows very easily from these facts and the explicit formula of the zeta functions. We can prove Theorem 4 by using Proposition 3.6.

\section{$\S 5$. Functional equation for $n=2$}

When $n=2$, we need some modification of the definition of our zeta functions since the volume $\mu(x)$ in the definition of zeta functions for $n \geq 3$ is infinite for some $x \in V$ when $n=2$. Shintani [21] has given a modified definition of the zeta function and has shown the functional equations for such zeta functions. Later, Sato [16] gave a more theoretical definition of Shintani's modification by using a zeta function of two variables which is naturally obtained by applying the general theory of the prehomogeneous vector spaces for $\left(\mathrm{GL}_{2} \times \mathrm{GL}_{1}, V \times \mathbb{C}^{2}\right)$, and also gave an alternative proof 
of the functional equation. Here we give the third alternative proof of Shintani's results above by using real analytic Eisenstein series of half-integral weight. More precisely, we give a natural modification of the definition from our standpoint, show the functional equation of our zeta functions, and give the location of poles and the principal parts of the Laurent expansion there. For the zeta function of definite symmetric matrices (i.e., $\zeta_{2}(s, L, 1,1)$ ), this approach has already been used by Sturm [23]. So the main point is the case for indefinite symmetric matrices (i.e. $\zeta(s, L,-1,-1))$. Our idea is as follows. When we fix an odd integer $k$, the functional equation of the Mellin transforms of Eisenstein series in the previous section is valid as a meromorphic function of $s$ and $\sigma$ for the whole $\mathbb{C}^{2}$ plane. Now we fix $k$ to be 1 throughout this section. Then each side of the functional equation between $\Phi_{\sigma, 1}$ and $\Psi_{\sigma, 1}$ has a pole at $\sigma=2$, and this is the reason that the original definition of our zeta function cannot be applied in the case $n=2$. In fact, most of the coefficients of the Dirichlet series $\Phi_{\sigma, 1}(s)$ and $\Psi_{\sigma, 1}(s)$ with respect to $s$ have definite values at $\sigma=2$, which form the main part of our zeta function of the prehomogeneous vector space, but the modified terms come from the coefficients which have a pole at $\sigma=2$. Even if they have a pole, each term of the Laurent expansions of $\Phi_{\sigma, 1}(s)$ and $\Psi_{\sigma, 1}(s)$ along $\sigma=2$ still satisfies the functional equation. So by comparing the constant terms of the Laurent expansion of both sides of the above functional equation associated with Eisenstein series at $\sigma=2$, we give a suitable modification of zeta functions and the functional equation at the same time. By the way, it is easy to see that both coefficients of $(\sigma-2)^{-1}$ in the Laurent expansions are

$$
C_{2,1} \zeta(2)^{-1} 2^{s-2} \pi^{1 / 2-s} \Gamma(s-1 / 2) \zeta(2 s-1)
$$

and this gives us nothing new. Now we define the main part of our zeta functions. Define four Dirichlet series

$$
\begin{aligned}
\xi_{+}^{M}(s) & =\zeta(2 s) \sum_{d=1}^{\infty} h(d) \log \left(\epsilon_{d}\right) d^{-s} \\
\xi_{+}^{*, M}(s) & =\zeta(2 s)\left(\sum_{d=1}^{\infty} \frac{h(4 d) \log \left(\epsilon_{4 d}\right)}{(4 d)^{s}}+2^{-2 s} \sum_{d=1}^{\infty} \frac{h(4 d+1) \log \left(\epsilon_{4 d+1}\right)}{(4 d+1)^{s}}\right) \\
\xi_{-}(s) & =2 \zeta(2 s) \sum_{d=1}^{\infty} h(-d) w_{-d}^{-1} d^{-s}
\end{aligned}
$$




$$
\xi_{-}^{*}(s)=2 \zeta(2 s)\left(\sum_{d=1}^{\infty} \frac{h(-4 d)}{w_{-4 d}(4 d)^{s}}+2^{-2 s} \sum_{d=1}^{\infty} \frac{h(-4 d+1)}{w_{-4 d+1}(4 d-1)^{s}}\right),
$$

where $h(d)$ is the class number in the narrow sense of the order $\mathcal{O}_{d}$ of discriminant $d$ in a quadratic field, $\epsilon_{d}$ is the fundamental unit of norm 1 of $\mathcal{O}_{d}$, and $w_{d}$ is the number of units of $\mathcal{O}_{d}$. When $d$ is a square or not a discriminant, we regard $h(d)=0$.

Then, by the classical class number formula, we can show that

$$
\begin{aligned}
\xi_{+}^{M}(s) & =\sum_{d_{K}>0, d_{K} \neq 1} \zeta(2) Z^{*}\left(1,2, s, d_{K}\right), \\
\pi \xi_{-}(s) & =\sum_{d_{K}<0} \zeta(2) Z^{*}\left(1,2, s, d_{K}\right) .
\end{aligned}
$$

Now we consider $Z(1, \sigma, s, 1)$ or $Z^{*}(1, \sigma, s, 1)$, the terms corresponding to $d_{K}=1$. If we write

$$
\frac{\zeta(2 s) \zeta(2 s-2 \sigma+3)}{\zeta(2 s-\sigma+2)}=\sum_{n=1}^{\infty} \frac{a(n)}{n^{s}}
$$

then we have

$$
\sum_{n=1}^{\infty} \frac{a(4 n)}{n^{s}}=\frac{\zeta(2 s) \zeta(2 s-2 \sigma+3)}{\zeta(2 s-\sigma+2)} \times \frac{1-2^{\sigma-2}+2^{2 \sigma-3}-2^{-2 s+2 \sigma-3}}{1-2^{-2 s+\sigma-2}} .
$$

So we put

$$
\begin{aligned}
h(\sigma, s)= & \frac{\zeta(2 s) \zeta(2 s-2 \sigma+3)}{\zeta(2 \sigma-2) \zeta(2 s-\sigma+2)} I(s, \sigma / 2,(\sigma-1) / 2) \\
h^{*}(\sigma, s)= & \frac{\zeta(2 s) \zeta(2 s-2 \sigma+3)}{\zeta(2 \sigma-2) \zeta(2 s-\sigma+2)} \frac{\left(1-2^{\sigma-2}+2^{2 \sigma-3}-2^{-2 s+2 \sigma-3}\right)}{\left(1-2^{-2 s+\sigma-2}\right)} \\
& \times I(s, \sigma / 2,(\sigma-1) / 2) .
\end{aligned}
$$

We define two functions $g(\sigma, s)$ and $g^{*}(\sigma, s)$ so that the following equalities hold:

$$
\begin{aligned}
& \Phi_{\sigma, 1}(s)=C_{\sigma, 1}(2 \pi)^{-s} \Gamma(s)(g(\sigma, s)+\zeta(\sigma-1) h(\sigma, s)), \\
& \Psi_{\sigma, 1}(s)=C_{\sigma, 1}(2 \pi)^{-s} \Gamma(s)\left(g^{*}(\sigma, s)+\zeta(\sigma-1) h^{*}(\sigma, s)\right) .
\end{aligned}
$$

Then, we have 


$$
\begin{aligned}
g(2, s) & =\zeta(2)^{-1}\left(\xi_{+}^{M}(s) I(s, 1,1 / 2)+\pi \xi_{-}(s) I(s, 1 / 2,1)\right) \\
g^{*}(2, s) & =2^{2 s} \zeta(2)^{-1}\left(\xi_{+}^{*, M}(s) I(s, 1,1 / 2)+\pi \xi_{-}^{*}(s) I(s, 1 / 2,1)\right)
\end{aligned}
$$

To obtain the functional equation which coincides with Shintani's original version (see [21]), it is convenient to multiply both sides of the functional equation between $\Phi_{\sigma, 1}(\sigma-s-1 / 2)$ and $\Psi_{\sigma, 1}(s)$ in Proposition 3.2 by

$$
\epsilon(\sigma, s)=C_{\sigma, 1}^{-1}(2 \pi)^{\sigma-s-1 / 2} 2^{(\sigma-1) / 2} \zeta(2 \sigma-2) \Gamma((\sigma-1) / 2)^{-1} \Gamma(1-s)^{-1} .
$$

To rewrite the functional equation in this form, we put

$$
\begin{aligned}
A(\sigma, s)= & \frac{2^{\frac{\sigma-1}{2}} \zeta(2 \sigma-2)}{\Gamma\left(\frac{\sigma-1}{2}\right)} \cdot \frac{\Gamma\left(\sigma-s-\frac{1}{2}\right)}{\Gamma(1-s)} g\left(\sigma, \sigma-s-\frac{1}{2}\right), \\
A^{*}(\sigma, s)= & \frac{2^{\frac{\sigma-1}{2}} \zeta(2 \sigma-2)}{\Gamma\left(\frac{\sigma-1}{2}\right)} \cdot 2^{-\sigma+1} \pi^{\sigma-2 s-1 / 2} \frac{\Gamma(s)}{\Gamma(1-s)} g^{*}(\sigma, s), \\
B(\sigma, s)= & \frac{\Gamma\left(\sigma-s-\frac{1}{2}\right)}{\Gamma\left(\frac{\sigma+1}{2}-s\right)} \frac{\zeta(2 \sigma-1-2 s) \zeta(2-2 s)}{\zeta(\sigma+1-2 s)} \\
& \times F_{2}(\sigma, s), \\
B^{*}(\sigma, s)= & 2^{-\sigma+1} \pi^{\sigma-2 s-1 / 2} \frac{\Gamma(s) \Gamma\left(s-\sigma+\frac{3}{2}\right)}{\Gamma(1-s) \Gamma\left(s-\frac{\sigma}{2}+1\right)} \\
& \times \frac{\zeta(2 s) \zeta(2 s-2 \sigma+3)}{\zeta(2 s-\sigma+2)} \times \frac{1-2^{\sigma-2}+2^{2 \sigma-3}-2^{-2 s+2 \sigma-3}}{1-2^{-2 s+\sigma-2}} \\
& \times F_{1}(\sigma, s),
\end{aligned}
$$

where we put

$$
\begin{aligned}
& F_{1}(\sigma, s)=F\left(1-\frac{\sigma}{2}, \frac{\sigma-1}{2} ; s-\frac{\sigma}{2}+1 ; \frac{1}{2}\right) \\
& F_{2}(\sigma, s)=F\left(1-\frac{\sigma}{2}, \frac{\sigma-1}{2}, \frac{\sigma+1}{2}-s ; \frac{1}{2}\right)
\end{aligned}
$$

Then we have

$$
\begin{aligned}
\epsilon(\sigma, s) \Phi_{\sigma, 1}\left(\sigma-s-\frac{1}{2}\right) & =A(\sigma, s)+B(\sigma, s) \zeta(\sigma-1), \\
2^{2 s-2 \sigma+3 / 2} \epsilon(\sigma, s) \Psi_{\sigma, 1}(s) & =A^{*}(\sigma, s)+\zeta(\sigma-1) B^{*}(\sigma, s) .
\end{aligned}
$$


So the functional equation in Proposition 3.2 reads

$$
A(\sigma, s)+B(\sigma, s) \zeta(\sigma-1)=A^{*}(\sigma, s)+\zeta(\sigma-1) B^{*}(\sigma, s) .
$$

The residue of each side at $\sigma=2$ is given by $B(2, s)$ or $B^{*}(2, s)$. But since $F_{1}(2, s)=F_{2}(2, s)=1$, we have $B(2, s)=\zeta(2-2 s)$ and $B^{*}(2, s)=$ $\pi^{3 / 2-2 s} \Gamma(s-1 / 2) \zeta(2 s-1) / \Gamma(1-s)$, so $B(2, s)=B^{*}(2, s)$ by the functional equation of the Riemann zeta function. Comparing the constant terms of the above functional equation at $\sigma=2$, we have

$$
\begin{aligned}
& A(2, s)+\left.\frac{d}{d \sigma} B(\sigma, s)\right|_{\sigma=2}+B(2, s) \gamma \\
& =A^{*}(2, s)+\left.\frac{d}{d \sigma} B^{*}(\sigma, s)\right|_{\sigma=2}+B^{*}(2, s) \gamma,
\end{aligned}
$$

where $\gamma$ is the Euler constant. As is well known, we have $\zeta(\sigma-1)=1 /(\sigma-$ $2)+\gamma+O(\sigma-2)$ and $\gamma=-\Gamma^{\prime}(1)$. Since we have $B(2, s)=B^{*}(2, s)$, the last terms cancel each other out. Since $I(s, 1,1 / 2)=\sqrt{\pi} \Gamma(s-1 / 2) / \sqrt{2} \Gamma(s)$, we can show easily that

$$
\begin{aligned}
A(2, s)= & \xi_{+}^{M}(3 / 2-s) \\
& +\sqrt{2 \pi} \frac{\Gamma(3 / 2-s)}{\Gamma(1-s)} \xi_{-}(3 / 2-s) I(3 / 2-s, 1 / 2,1) \\
A^{*}(2, s)= & 2^{2 s-1} \pi^{1 / 2-2 s} \Gamma(s) \Gamma(s-1 / 2) \\
& \times\left(\sin (\pi s) \xi_{+}^{*, M}(s)+\pi^{3 / 2} \sqrt{2} \frac{\xi_{-}^{*}(s)}{\Gamma(s-1 / 2) \Gamma(1-s)} I(s, 1 / 2,1)\right) .
\end{aligned}
$$

It was proved in Section 3.2 that

$$
\begin{aligned}
\xi_{-}(3 / 2-s)= & 2^{2 s-1} \pi^{-2 s+1 / 2} \Gamma(s) \Gamma(s-1 / 2) \cos (\pi s) \xi_{-}^{*}(s) \\
& -2^{-1} \pi^{-2 s+1 / 2} \Gamma(s) \Gamma(s-1 / 2) \zeta(2 s-1)
\end{aligned}
$$

Using this functional equation and the well-known fact that

$$
\cos (\pi s)=-\pi / \Gamma(s-1 / 2) \Gamma(3 / 2-s),
$$

we have

$$
\begin{aligned}
A(2, s)= & \xi_{+}^{M}(3 / 2-s) \\
& -2^{2 s-1 / 2} \pi^{2-2 s} \Gamma(s) \Gamma(1-s)^{-1} \xi_{-}^{*}(s) I(3 / 2-s, 1 / 2,1)
\end{aligned}
$$




$$
\begin{aligned}
& -2^{-1 / 2} \pi^{1-2 s} \Gamma(s) \Gamma(s-1 / 2) \Gamma(3 / 2-s) \Gamma(1-s)^{-1} \\
& \times \zeta(2 s-1) I(3 / 2-s, 1 / 2,1) .
\end{aligned}
$$

Hence, by using the formula

$$
I(s, 1 / 2,1)+I(3 / 2-s, 1 / 2,1)=\frac{\Gamma(1-s) \Gamma(s-1 / 2)}{\sqrt{2 \pi}}
$$

and applying the functional equation, we have

$$
\begin{aligned}
\xi_{+}^{M}(3 / 2-s)+\left.\frac{d}{d \sigma} B(\sigma, s)\right|_{\sigma=2} \\
=2^{2 s-1} \pi^{-2 s+1 / 2} \Gamma(s) \Gamma(s-1 / 2)\left\{\sin (\pi s) \xi_{+}^{*, M}(s)+\pi \xi_{-}^{*}(s)\right\} \\
\quad+\frac{\pi^{1-2 s}}{\sqrt{2}} \frac{\Gamma(s) \Gamma(s-1 / 2) \Gamma(3 / 2-s)}{\Gamma(1-s)} \\
\quad \times \zeta(2 s-1) I\left(\frac{3}{2}-s, \frac{1}{2}, 1\right)+\left.\frac{d}{d \sigma} B^{*}(\sigma, s)\right|_{\sigma=2} .
\end{aligned}
$$

In order to write the functional equation more explicitly, we must calculate the difference

$$
\left.\frac{d}{d \sigma} B^{*}(\sigma, s)\right|_{\sigma=2}-\left.\frac{d}{d \sigma} B(\sigma, s)\right|_{\sigma=2}
$$

When we differentiate $B(\sigma, s)$ and $B^{*}(\sigma, s)$, we regard these roughly as products of functions of $\sigma$ which are powers of 2 , the shifted Riemann zeta functions, gamma factors, and hypergeometric functions. We have

$$
\begin{aligned}
\left.\frac{d}{d \sigma} B(\sigma, s)\right|_{\sigma=2}= & \zeta(2-2 s)\left(\frac{\zeta^{\prime}(3-2 s)}{\zeta(3-2 s)}-\frac{\zeta^{\prime}(2-2 s)}{\zeta(2-2 s)}\right)+\zeta^{\prime}(2-2 s) \\
& +\frac{1}{2} \zeta(2-2 s) \frac{\Gamma^{\prime}(3 / 2-s)}{\Gamma(3 / 2-s)} \\
& +\left.\zeta(2-2 s) \frac{d}{d \sigma} F_{2}(\sigma, s)\right|_{\sigma=2}
\end{aligned}
$$

and

$$
\begin{aligned}
& \left.\frac{d}{d \sigma} B^{*}(\sigma, s)\right|_{\sigma=2} \\
& \quad=\pi^{3 / 2-2 s} \frac{\Gamma(s-1 / 2)}{\Gamma(1-s)}\left(\zeta(2 s-1)\left(\frac{\zeta^{\prime}(2 s)}{\zeta(2 s)}-\frac{\zeta^{\prime}(2 s-1)}{\zeta(2 s-1)}\right)-\zeta^{\prime}(2 s-1)\right)
\end{aligned}
$$




$$
\begin{aligned}
& +(\log \pi) \pi^{3 / 2-2 s} \zeta(2 s-1) \frac{\Gamma(s-1 / 2)}{\Gamma(1-s)} \\
& +(\log 2) \pi^{3 / 2-2 s} \frac{\Gamma(s-1 / 2)}{\Gamma(1-s)} \frac{\zeta(2 s-1)}{2\left(1-2^{-2 s}\right)} \\
& +2^{-1} \pi^{1 / 2-2 s} \zeta(2 s-1) \Gamma(s) \Gamma(s-1 / 2)(\sin \pi s)\left(\frac{\Gamma^{\prime}(s)}{\Gamma(s)}-\frac{\Gamma^{\prime}(s-1 / 2)}{\Gamma(s-1 / 2)}\right) \\
& -2^{-1} \pi^{1 / 2-2 s} \zeta(2 s-1) \Gamma(s) \Gamma(s-1 / 2)(\sin \pi s) \frac{\Gamma^{\prime}(s-1 / 2)}{\Gamma(s-1 / 2)} \\
& +\left.\pi^{3 / 2-2 s} \zeta(2 s-1) \frac{\Gamma(s-1 / 2)}{\Gamma(1-s)} \frac{d}{d \sigma} F_{1}(\sigma, s)\right|_{\sigma=2} .
\end{aligned}
$$

By the functional equation of the Riemann zeta function and the well-known formula $\Gamma(s) \Gamma(1-s)=\pi / \sin (\pi s)$, we have

$$
\begin{aligned}
\zeta(2-2 s) & =\pi^{3 / 2-2 s} \zeta(2 s-1) \frac{\Gamma(s-1 / 2)}{\Gamma(1-s)} \\
& =\pi^{1 / 2-2 s}(\sin \pi s) \zeta(2 s-1) \Gamma(s) \Gamma(s-1 / 2) .
\end{aligned}
$$

Now we will calculate

$$
\left.\zeta(2-2 s) \frac{d}{d \sigma}\left(F_{1}(\sigma, s)-F_{2}(\sigma, s)\right)\right|_{\sigma=2} .
$$

The following formula of the hypergeometric functions is well known:

$$
\begin{aligned}
F(a, b ; c, z)= & \frac{\Gamma(c) \Gamma(c-a-b)}{\Gamma(c-a) \Gamma(c-b)} F(a, b ; a+b-c+1 ; 1-z) \\
& +(1-z)^{c-a-b} \frac{\Gamma(c) \Gamma(a+b-c)}{\Gamma(a) \Gamma(b)} \\
& \times F(c-a, c-b ; c-a-b+1 ; 1-z) .
\end{aligned}
$$

So, we have

$$
\begin{aligned}
F_{1}(\sigma, s)= & \frac{\Gamma(s-\sigma / 2+1) \Gamma(s-(\sigma-1) / 2)}{\Gamma(s) \Gamma(s-\sigma+3 / 2)} F_{2}(\sigma, s) \\
& +2^{-s+(\sigma-1) / 2} \frac{\Gamma(s-\sigma / 2+1) \Gamma(-s+(\sigma-1) / 2)}{\Gamma(1-\sigma / 2) \Gamma((\sigma-1) / 2)} \\
& \times F\left(s, s-\sigma+3 / 2 ; s-(\sigma-3) / 2 ; \frac{1}{2}\right) .
\end{aligned}
$$


Now, we differentiate at $\sigma=2$ both sides of the above formula. Since $\Gamma(1-$ $\sigma / 2)^{-1}$ has a simple zero at $\sigma=2$ and $\left.(d / d \sigma) \Gamma(1-\sigma / 2)^{-1}\right|_{\sigma=2}=-1 / 2$, the differential coefficient at $\sigma=2$ of the second term of the right-hand side is

$$
-2^{-1 / 2-s} \frac{\Gamma(s) \Gamma(1 / 2-s)}{\Gamma(1 / 2)} F(s, s-1 / 2 ; s+1 / 2 ; 1 / 2),
$$

but we see that

$$
\begin{aligned}
F\left(s, s-\frac{1}{2} ; s+\frac{1}{2} ; \frac{1}{2}\right) & =\frac{\Gamma\left(s+\frac{1}{2}\right)}{\Gamma\left(s-\frac{1}{2}\right)} \int_{0}^{1} t^{s-3 / 2}\left(1-\frac{1}{2} t\right)^{-s} d t \\
& =2^{s-1 / 2} \frac{\Gamma\left(s+\frac{1}{2}\right)}{\Gamma\left(s-\frac{1}{2}\right)} \int_{1 / 2}^{1}(1-v)^{s-3 / 2} v^{-s} d t \\
& =2^{s} \frac{\Gamma\left(s+\frac{1}{2}\right)}{\Gamma\left(s-\frac{1}{2}\right)} I\left(s, \frac{1}{2}, 1\right) .
\end{aligned}
$$

Hence, we have

$$
\begin{aligned}
\left.\frac{d}{d \sigma}\left(F_{1}-F_{2}\right)\right|_{\sigma=2}= & -\frac{1}{2}\left(\frac{\Gamma^{\prime}(s)}{\Gamma(s)}-\frac{\Gamma^{\prime}(s-1 / 2)}{\Gamma(s-1 / 2)}\right) \\
& -(2 \pi)^{-1 / 2} \frac{\Gamma(s) \Gamma(1 / 2-s) \Gamma(s+1 / 2)}{\Gamma(s-1 / 2)} I(s, 1 / 2,1) .
\end{aligned}
$$

Using the notation of Shintani [21], we define

$$
\begin{aligned}
\xi_{+}(s)= & \xi_{+}^{M}(s)+\zeta(2 s-1)\left(\frac{\zeta^{\prime}(2 s)}{\zeta(2 s)}-\frac{\zeta^{\prime}(2 s-1)}{\zeta(2 s-1)}\right) \\
\xi_{+}^{*}(s)= & \xi_{+}^{*, M}(s)+2^{1-2 s} \zeta(2 s-1)\left(\frac{\zeta^{\prime}(2 s)}{\zeta(2 s)}-\frac{\zeta^{\prime}(2 s-1)}{\zeta(2 s-1)}\right) \\
& +2^{-2 s}(\log 2)\left(1-2^{-2 s}\right)^{-1} \zeta(2 s-1) .
\end{aligned}
$$

We prepare two more relations. By differentiating the usual functional equation of the Riemann zeta function, we have

$$
\frac{\zeta^{\prime}(2-2 s)}{\zeta(2-2 s)}=\log \pi-\frac{1}{2} \frac{\Gamma^{\prime}(s-1 / 2)}{\Gamma(s-1 / 2)}-\frac{1}{2} \frac{\Gamma^{\prime}(1-s)}{\Gamma(1-s)}-\frac{\zeta^{\prime}(2 s-1)}{\zeta(2 s-1)} .
$$

On the other hand, by differentiating the relations $\Gamma(s) \Gamma(1-s)=\pi / \sin \pi s$ and $\Gamma(s-1 / 2) \Gamma(3 / 2-s)=-\pi / \cos \pi s$, we have

$$
\frac{\Gamma^{\prime}(s-1 / 2)}{\Gamma(s-1 / 2)}+\frac{\Gamma^{\prime}(1-s)}{\Gamma(1-s)}=\frac{\pi}{(\cos \pi s)(\sin \pi s)}+\frac{\Gamma^{\prime}(s)}{\Gamma(s)}+\frac{\Gamma^{\prime}(3 / 2-s)}{\Gamma(3 / 2-s)} .
$$


By comparing all the equalities we obtained above, we have the alternative proof of the functional equation in Proposition 5.1 below. This was first obtained by Shintani [21] by using Dirichlet series of two variables attached to quadratic forms. These Dirichlet series of two variables are natural objects corresponding to the theory of quadratic forms, but his method of the proof itself is somewhat more technical and not necessarily easy to understand conceptually.

Proposition 5.1 (Shintani [21], Sato [16]). Two zeta functions $\xi_{+}(s)$ and $\xi_{+}^{*}(s)$ are meromorphically continued to the whole $s$ plane and satisfy the following functional equation:

$$
\begin{aligned}
\xi_{+} & (3 / 2-s) \\
= & 2^{2 s-1} \pi^{-2 s+1 / 2} \Gamma(s) \Gamma(s-1 / 2)\left((\sin \pi s) \xi_{+}^{*}(s)+\pi \xi_{-}^{*}(s)\right) \\
& +2^{-1} \pi^{-2 s+1 / 2} \Gamma(s) \Gamma(s-1 / 2) \zeta(2 s-1)(\sin \pi s)\left(\frac{\Gamma^{\prime}(s)}{\Gamma(s)}-\frac{\Gamma^{\prime}(s-1 / 2)}{\Gamma(s-1 / 2)}\right) .
\end{aligned}
$$

Next, for the sake of completeness, we will calculate the principal part of the Laurent expansion at poles of $\xi_{+}(s)$ and $\xi_{+}^{*}(s)$ by our method. For that purpose, we need more precise information on the location of poles and their residues of the functions which appear in $\Phi_{\sigma, 1}(s)$ and $\Psi_{\sigma, 1}(s)$.

We define

$$
I_{2}(s)=\frac{\Gamma(3 / 2-s)}{2 \sqrt{\pi} \Gamma(1-s)} \int_{0}^{1} u^{-1 / 2}(1-u)^{-s} \log (1-u / 2) d u .
$$

This function converges when $\operatorname{Re}(s)<1$. Differentiating the integral expression of the beta function

$$
B\left(t+\frac{1}{2}, 1-s\right)=\frac{\Gamma(t+1 / 2) \Gamma(1-s)}{\Gamma(t+3 / 2-s)}=\int_{0}^{1} u^{t-1 / 2}(1-u)^{-s} d u
$$

at $t=0$ under the integral sign, we have

$$
\begin{aligned}
& \frac{\Gamma(3 / 2-s)}{\sqrt{\pi} \Gamma(1-s)} \int_{0}^{1} u^{-1 / 2}(1-u)^{-s} \log (u) d u \\
& =\frac{\Gamma^{\prime}(1 / 2) \Gamma(3 / 2-s)-\Gamma(1 / 2) \Gamma^{\prime}(3 / 2-s)}{\sqrt{\pi} \Gamma(3 / 2-s)} .
\end{aligned}
$$

Using this relation, and differentiating under the integral sign, we have

$$
\left.\frac{d}{d \sigma}\left(F_{2}(\sigma, s)\right)\right|_{\sigma=2}=I_{2}(s)
$$




$$
\left.\frac{d}{d \sigma}\left(F_{1}(\sigma, s)\right)\right|_{\sigma=2}=I_{2}\left(\frac{3}{2}-s\right)
$$

As before, for a nonnegative integer $n$ and a variable number $a$, we put $(a)_{n}=\Gamma(a+n) / \Gamma(a)$. We define the Barnes generalized hypergeometric function ${ }_{3} F_{2}$ as usual by

$$
{ }_{3} F_{2}\left(\alpha_{1}, \alpha_{2}, \alpha_{3} ; \beta_{1}, \beta_{2} ; z\right)=\sum_{n=0}^{\infty} \frac{\left(\alpha_{1}\right)_{n}\left(\alpha_{2}\right)_{n}\left(\alpha_{3}\right)_{n}}{\left(\beta_{1}\right)_{n}\left(\beta_{2}\right)_{n}} \frac{z^{n}}{n !} .
$$

Then, we also have

$$
\begin{aligned}
I_{2}(s) & =-\sum_{n=1}^{\infty} \frac{1}{2^{n+1} n} \frac{\Gamma(n+1 / 2) \Gamma(3 / 2-s)}{\sqrt{\pi} \Gamma(n+3 / 2-s)} \\
& =-\frac{\Gamma(3 / 2-s)}{2^{3} \Gamma(5 / 2-s)}{ }_{3} F_{2}(1,1,3 / 2 ; 2,5 / 2-s ; 1 / 2) .
\end{aligned}
$$

Since $I_{2}(s)$ is holomorphic for $\operatorname{Re}(s)<1$ and $I_{2}(3 / 2-s)$ for $\operatorname{Re}(s)>1 / 2$, $I_{2}(s)$ is meromorphically continued to the whole $s$ plane by virtue of the formula for $I_{2}(3 / 2-s)-I_{2}(s)$ that we gave before. It is also easy to show that $I_{2}(s)$ has simple poles at $s=1 / 2+m(m=1,2, \ldots)$ with residues

$$
(-1)^{m+1} 2^{-1 / 2-2 m}(2 m) !(m !)^{-2} m I(1 / 2+m, 1 / 2,1)
$$

and that it is holomorphic for all other $s$. We have

$$
\begin{aligned}
\left.\frac{d}{d \sigma} B(\sigma, s)\right|_{\sigma=2}= & \zeta(2-2 s)\left(\frac{\zeta^{\prime}(3-2 s)}{\zeta(3-2 s)}-\frac{\zeta^{\prime}(2-2 s)}{\zeta(2-2 s)}\right)+\zeta^{\prime}(2-2 s) \\
& +\frac{1}{2} \zeta(2-2 s) \frac{\Gamma^{\prime}(3 / 2-s)}{\Gamma(3 / 2-s)}+\zeta(2-2 s) I_{2}(s) .
\end{aligned}
$$

We write $U_{r}(a)=\{\sigma \in \mathbb{C} ;|\sigma-a|<r\}$. Then it was shown in Section 3 that the function

$$
(\sigma-2) s(s-\sigma+1 / 2)(s-\sigma+3 / 2) \epsilon(\sigma, s) \Phi_{\sigma, 1}(\sigma-1 / 2-s)
$$

is holomorphic on $(\sigma, s) \in U_{3 / 2}(2) \times \mathbb{C}$. Now, notation being as in Section 3, we put 


$$
\begin{aligned}
\widetilde{J}(\sigma, s)= & 2^{s+1 / 2-\sigma} \epsilon(\sigma, s) \\
& \times\left(\int_{1}^{\infty}\left(G_{1}(y / 2)-c_{0}(y / 2)\right) y^{\sigma-3 / 2-s} d y\right. \\
& \left.+C_{1} \int_{1}^{\infty}\left(H_{1}(y / 2)-c_{0}(y / 8)\right) y^{s-1} d y\right) .
\end{aligned}
$$

Then, $(\sigma-2) \Gamma(1-s) \widetilde{J}(\sigma, s)$ is holomorphic on $(\sigma, s) \in U_{3 / 2}(2) \times \mathbb{C}$. Using the Hecke-type integral expression of $\Phi_{\sigma, k}(s)$ obtained in Section 3 for $k=1$, we have

$$
\begin{aligned}
& A(\sigma, s)+\zeta(\sigma-1) B(\sigma, s) \\
& \quad=\widetilde{J}(\sigma, s)-\pi^{1-s} \frac{\Gamma(\sigma-3 / 2) \zeta(2 \sigma-3)}{\Gamma((\sigma-1) / 2) \Gamma(1-s)} \times\left(\frac{2^{\sigma / 2-1}}{s-\sigma+3 / 2}+\frac{2^{1-\sigma / 2}}{1-s}\right) \\
& -\pi^{-s} \frac{\Gamma(\sigma / 2) \zeta(2 \sigma-2)}{\Gamma(1-s)} \times\left(\frac{2^{(\sigma-3) / 2}}{s}+\frac{2^{(3 \sigma-5) / 2}}{\sigma-1 / 2-s}\right) .
\end{aligned}
$$

We compare the constant term of the Laurent expansion at $\sigma=2$ of the above equality. We write the Laurent expansion of $\widetilde{J}(\sigma, s)$ at $\sigma=2$ as $J_{-1}(s) /(\sigma-2)+J_{0}(s)+O(\sigma-2)$. Here $J_{-1}(s)$ and $J_{0}(s)$ are entire functions of $s$. Then the constant term of the left-hand side is given by

$$
\begin{aligned}
f(s)= & A(2, s)+\left.\frac{d}{d \sigma} B(\sigma, s)\right|_{\sigma=2}+\gamma B(2, s) \\
= & \xi_{+}(3 / 2-s) \\
& +\sqrt{2 \pi} \frac{\Gamma(3 / 2-s)}{\Gamma(1-s)} \xi_{-}(3 / 2-s) I(3 / 2-s, 1 / 2,1)+\zeta^{\prime}(2-2 s) \\
& +\frac{1}{2} \zeta(2-2 s) \frac{\Gamma^{\prime}(3 / 2-s)}{\Gamma(3 / 2-s)}+\zeta(2-2 s) I_{2}(s)+\gamma \zeta(2-2 s) .
\end{aligned}
$$

We calculate the constant term $g(s)$ of the right-hand side. We have

$$
\begin{aligned}
\zeta(2 \sigma-3) & =\frac{1}{2(\sigma-2)}+\gamma+O(\sigma-2) \\
\frac{1}{s-\sigma+3 / 2} & =(s-1 / 2)^{-1}+(s-1 / 2)^{-2}(\sigma-2)+O\left((\sigma-2)^{2}\right) \\
2^{\sigma / 2-1} & =1+\frac{1}{2} \log (2)(\sigma-2)+O\left((\sigma-2)^{2}\right)
\end{aligned}
$$




$$
\frac{\Gamma(\sigma-3 / 2)}{\Gamma((\sigma-1) / 2)}=1+\frac{\Gamma^{\prime}(1 / 2)}{2 \Gamma(1 / 2)}(\sigma-2)+O\left((\sigma-2)^{2}\right) .
$$

Here it is well known that $\Gamma(1 / 2)=\sqrt{\pi}$ and that $\Gamma^{\prime}(1 / 2) / \Gamma(1 / 2)=-\gamma-$ $2 \log (2)$. Hence, we have

$$
\begin{aligned}
g(s)= & J_{0}(s)-\pi^{-s} \frac{\zeta(2)}{\Gamma(1-s)}\left(\frac{1}{\sqrt{2} s}+\frac{\sqrt{2}}{3 / 2-s}\right) \\
& -\frac{\pi^{1-s}}{\Gamma(1-s)(1-s)}\left(\frac{\Gamma^{\prime}(1 / 2)}{4 \Gamma(1 / 2)}-\frac{1}{4} \log (2)+\gamma\right) \\
& -\frac{\gamma \pi^{1-s}}{\Gamma(1-s)(s-1 / 2)}+\frac{1}{2}\left(\frac{\pi^{1-s}(\gamma+\log (2))}{2(s-1 / 2) \Gamma(1-s)}-\frac{\pi^{1-s}}{(s-1 / 2)^{2} \Gamma(1-s)}\right) .
\end{aligned}
$$

We have $f(s)=g(s)$ by definition. We compare the principal part of the Laurent expansion of $f(s)=g(s)$ at $s=1 / 2$. First, we see that the order of the pole of $\xi_{+}(3 / 2-s)$ at $s=1 / 2$ is at most 2 , so we write the principal part of the Laurent expansion of $\xi_{+}(s)$ at $s=1$ by $\xi_{+}(s)=a_{-2}(s-1)^{-2}+$ $a_{-1}(s-1)^{-1}+O(1)$. Then we have $\xi_{+}(3 / 2-s)=a_{-2}(s-1 / 2)^{-2}-a_{-1}(s-$ $1 / 2)^{-1}+O(1)$. We compare the coefficients of $(s-1 / 2)^{-2}$. Then, since

$$
\zeta^{\prime}(2-2 s)=-\frac{1}{4}(s-1 / 2)^{-2}+\gamma+O(s-1 / 2),
$$

we have $a_{-2}-1 / 4=-1 / 2$ and $a_{-2}=-1 / 4$. Next, we compare the residues at $s=1 / 2$. Using the values $I(1,1 / 2,1)=\sqrt{2} \log (\sqrt{2}+1)$ and $I_{2}(1 / 2)=$ $-2^{-1} 3 \log (2)+\log (\sqrt{2}+1)$ and the fact that $\xi_{-}(3 / 2-s)=4^{-1}(s-1 / 2)^{-1}+$ $O(1)$, we see that the residue of $f(s)$ at $s=1 / 2$ is given by

$$
-a_{-1}-\frac{1}{4} \gamma+\frac{3}{4} \log (2)
$$

Since we have

$$
\frac{\pi^{1-s}}{\Gamma(1-s)}=1+\left(\frac{\Gamma^{\prime}(1 / 2)}{\Gamma(1 / 2)}-\log (\pi)\right)\left(s-\frac{1}{2}\right)+O\left(\left(s-\frac{1}{2}\right)^{2}\right),
$$

the residue of $g(s)$ at $s=1 / 2$ is

$$
\begin{aligned}
-\gamma & +\frac{1}{2}\left(\frac{\gamma}{2}+\frac{1}{2} \log (2)-(-\gamma-2 \log (2)-\log (\pi))\right) \\
= & -\frac{1}{4} \gamma+\frac{5}{4} \log (2)+\frac{1}{2} \log (\pi) .
\end{aligned}
$$


Hence, we have $a_{-1}=-2^{-1} \log (2 \pi)$.

Next, we see the principal part around $s=3 / 2$. We denote the residue of $\xi_{+}(s)$ at $s=3 / 2$ by $d$. Since $\xi_{-}(3 / 2-s)=-\pi /(12 s)+\cdots$ and $I(3 / 2,1 / 2,1)=$ $2-\sqrt{2}$, comparing the residues of $f(s)$ and $g(s)$ at $s=0$, we see that $-d-$ $(\sqrt{2})^{-1}\left(\pi^{2} / 12\right)(2-\sqrt{2})=-\pi^{2} / 6 \sqrt{2}$. So we have $d=\pi^{2} / 12$.

Now, we will show that $\xi_{+}(s)$ is holomorphic for all $s \neq 1,3 / 2$. Since $\Gamma(1-s)^{-1} I(3 / 2-s, 1 / 2,1)$ is entire, and since $I_{2}(s)$ has poles only at $s=$ $1 / 2+m(m=1,2, \ldots)$, we see that $\xi_{+}(3 / 2-s)$ is holomorphic for $s \neq 0,1 / 2$, $1 / 2+m$. Since the residue of $\Gamma(1 / 2-s)$ at $s=m+1 / 2$ is $(-1)^{m} / \Gamma(m+1)$, we see that the residue at $s=m+1 / 2$ of $I_{2}(s) / 2$ is

$$
(-1)^{m} 2^{-2} \pi^{-1} \Gamma(m)^{-1} \Gamma(m+1 / 2) I(1 / 2+m, 1 / 2,1) .
$$

The residue of $\Gamma^{\prime}(3 / 2-s) / \Gamma(3 / 2-s)$ at $m+1 / 2$ is $-1 / 2$. By the functional equation for $\xi_{-}(s)$, we see that $\xi_{-}(1-m)=-2^{-1}(-1)^{m} \zeta(1-2 m)$ for positive integers $m \geq 2$ and $\xi_{-}(0)=2^{-1} \zeta(-1)+1 / 12$. We have $I(1-m, 1 / 2,1)+$ $I(m+1 / 2,1 / 2,1)=(2 \pi)^{-1} \Gamma(1 / 2-m) \Gamma(m)$ and $I(3 / 2,1 / 2,1)=2-\sqrt{2}$, $I(0,1 / 2,1)=-2$. So, comparing the Laurent expansions at $s=m+1 / 2$ of $f(s)$ and $g(s)$, it is easy to see that $\xi_{+}(3 / 2-s)$ is holomorphic also at $s=m+1 / 2$ for positive integers $m$. Thus, we obtained all the locations of poles of $\xi_{+}(s)$ and its principal part of the Laurent expansion at each pole. For $\xi_{+}^{*}(s)$, if we denote by $f^{*}(s)$ the constant term of the Laurent expansion of $A^{*}(\sigma, s)+\zeta(\sigma-1) B^{*}(\sigma, s)$ at $\sigma=2$, then we have

$$
\begin{aligned}
2^{1-2 s} & \pi^{2 s-3 / 2} \Gamma(1-s) \Gamma(s-1 / 2)^{-1} f^{*}(s) \\
= & \xi_{+}^{*}(s)+(2 \pi)^{1 / 2} \frac{\Gamma(s)}{\Gamma(s-1 / 2)} \xi_{-}^{*}(s) I(s, 1 / 2,1) \\
& -2^{1-2 s} \zeta^{\prime}(2 s-1)+2^{1-2 s} \log (\pi) \zeta(2 s-1)+2^{1-2 s} \gamma \zeta(2 s-1) \\
& +2^{-2 s} \zeta(2 s-1)\left(\frac{\Gamma^{\prime}(s)}{\Gamma(s)}-2 \frac{\Gamma^{\prime}(s-1 / 2)}{\Gamma(s-1 / 2)}\right) \\
& +2^{1-2 s} \zeta(2 s-1) \times I_{2}(3 / 2-s) .
\end{aligned}
$$

By the functional equation, we have $\xi_{-}^{*}(1-m)=(-1)^{m+1} 2^{2 m-2} \zeta(1-2 m)$ for $m=2,3, \ldots$ and $\xi_{-}^{*}(0)=0$. Using this and comparing with the integral expression of the Hecke type in Section 3, we have the location of poles and the expansion of $\xi_{+}^{*}(s)$ at poles almost in the same way as before. We omit the details here. Thus, we have the alternative proof of the following results first obtained by Shintani [21] and Sato [16]. 
Proposition 5.2. Both zeta functions $\xi_{+}(s)$ and $\xi_{+}^{*}(s)$ are holomorphic except for $s=1$ and $s=3 / 2$. The principal part of the Laurent expansions are given as follows. At $s=1$, we have $\xi_{+}(s)=-4^{-1}(s-1)^{-2}-$ $2^{-1} \log (2 \pi)(s-1)^{-1}+\cdots$ and $\xi_{+}^{*}(s)=-8^{-1}(s-1)^{-2}-4^{-1} \log (2 \pi)(s-$ $1)^{-1}+\cdots$. At $s=3 / 2$, we have $\xi_{+}(s)=12^{-1} \pi^{2}(s-3 / 2)+\cdots$ and $\xi_{+}^{*}(s)=$ $24^{-1} \pi^{2}(s-3 / 2)^{-1}+\cdots$.

\section{$\S 6$. Special values of zeta functions and dimensions of Siegel cusp forms}

The values $\zeta\left(1-m, L_{n}^{*}, 1,1\right)$ for positive integers $m$ are related with dimension formulas of Siegel cusp forms and are important. In this section, first we give the formulas for the values of $\zeta(s, L, 1,1)$ at nonpositive integers for $L=L_{n}^{*}$ or $L_{n}$. These are rational and are described by Bernoulli numbers explicitly. Second, we apply these results to the dimension formulas of Siegel cusp forms. The results in this section have been announced in [6], but we reproduce them with more detailed explanation and the proof.

For each nonnegative integer $m$, we denote by $B_{m}$ the Bernoulli number defined by

$$
\frac{t e^{t}}{e^{t}-1}=\sum_{m=0}^{\infty} B_{m} \frac{t^{m}}{m !} .
$$

Now, we will give special values of the above zeta functions.

THEOREM 5. When $n$ is odd (including the case $n=1$ ), for each positive integer number $m$, we have

$$
\begin{gathered}
\zeta\left(1-m, L_{n}^{*}, 1,1\right) \\
=\frac{(-1)^{(n+1) / 2}\left|B_{2} B_{4} \cdots B_{n-1}\right| B_{m+\frac{n-1}{2}} B_{2 m} B_{2 m+2} \cdots B_{2 m+n-3}}{2^{(2 m+1)(n-1) / 2}\left(\frac{n-1}{2}\right) ! m(m+1) \cdots\left(m+\frac{n-1}{2}\right)}, \\
\zeta\left(1-m, L_{n}, 1,1\right)=2^{(2 m-1)(n-1) / 2} \zeta\left(1-m, L_{n}^{*}, 1,1\right) .
\end{gathered}
$$

TheOREM 6. When $n$ is even, for each positive integer $m$ with $n m \neq 2$, we have

$$
\begin{aligned}
\zeta\left(1-m, L_{n}^{*}, 1,1\right) & =\frac{(-1)^{\left[\frac{n}{4}\right]+n m / 2}\left|B_{2} B_{4} \cdots B_{n-2} B_{n / 2}\right| B_{2 m} B_{2 m+2} \cdots B_{2 m+n-2}}{2^{n m+(n-2) / 2}\left(\frac{n}{2}\right) ! m(m+1) \cdots\left(m+\frac{n-2}{2}\right)}, \\
\zeta\left(1-m, L_{n}, 1,1\right) & =2^{n m-n / 2} \zeta\left(1-m, L_{n}^{*}, 1,1\right) .
\end{aligned}
$$


When $n m=2$, which means that $(n, m)=(2,1)$, we have $\zeta\left(0, L_{2}^{*}, 1,1\right)=$ $1 / 48, \zeta\left(0, L_{2}, 1,1\right)=0$. In particular, if $n \equiv 2 \bmod 4$ and $n>2$, then $\zeta(1-$ $\left.m, L_{n}^{*}, 1,1\right)=\zeta\left(1-m, L_{n}, 1,1\right)=0$.

Proofs of Theorems 5 and 6 . The case when $n$ is odd is obvious, noting the well-known relation $\zeta(1-m)=-B_{m} / m$ for the Riemann zeta function. Now, assume that $n$ is even. By Propositions 3.3 and 3.4, $D_{n}^{*}(s, 1)$ and $D_{n}(s, 1)$ are holomorphic at $1-m(m=1,2, \ldots)$. When $n=2$, we have $\zeta\left(s, L_{2}^{*}, 1,1\right)=2^{2 s-1} D_{2}^{*}(s, 1)$ and $\zeta\left(s, L_{2}, 1,1\right)=2^{-1} D_{2}(s, 1)$. So, our results easily follow from Proposition 3.4. When $n \geq 4$, using notation as in Section 1 , we have $A_{n}(1-m)=0$ and

$$
B_{n}(1-m)=(-1)^{n / 2} \prod_{i=1}^{n / 2} B_{2 m+2 i-2} /(2 m+2 i-2),
$$

and we obtain the special values.

REMARK. When $n=2$, these values have been essentially known by the functional equation. When $n=3$, as a special case of the above results, we have $\zeta\left(0, L_{3}^{*}\right)=1 / 3456$. This value has been obtained by an indirect method by Hashimoto and Tsushima. They compared two complicated expressions of the dimension of Siegel cusp forms of degree 3, one by the Selberg trace formula and the other by the Riemann-Roch theorem.

Next, we will give explicit values of the contribution of "central" unipotent elements to the dimension of Siegel cusp forms. For any positive integers $n$ and $N$, we denote by $\Gamma_{n}(1)=\operatorname{Sp}(n, \mathbb{Z})$ the full Siegel modular group of size $2 n$ and by $\Gamma_{n}(N)$ the principal congruence subgroup of level $N$ of degree $n$ defined as usual by

$$
\Gamma_{n}(N)=\left\{g \in \operatorname{Sp}(n, \mathbb{Z}) ; g \equiv 1_{2 n} \bmod N\right\}
$$

For a positive integer $k$, we denote by $S_{k}\left(\Gamma_{n}(N)\right)$ the space of Siegel cusp forms of weight $k$ belonging to $\Gamma_{n}(N)$. First, we review the Godement dimension formula.

TheOREM 7 (Godement). For any integer $k>2 n$, we have

$$
\operatorname{dim} S_{k}\left(\Gamma_{n}(N)\right)=\frac{a_{n}(k)}{\# Z\left(\Gamma_{n}\right)} \int_{\Gamma_{n} \backslash H_{n}} \sum_{\gamma \in \Gamma_{n}} H_{\gamma}(Z) d Z
$$


where $d Z=(\operatorname{det}(Y))^{-n-1} d X d Y$,

$$
H_{\gamma}(Z)=\operatorname{det}\left(\frac{Z-\gamma \bar{Z}}{2 i}\right)^{-k} \operatorname{det}(C \bar{Z}+D)^{-k}(\operatorname{det} Y)^{k},
$$

and

$$
\begin{aligned}
a_{n}(k) & =\frac{1}{2^{n}(2 \pi)^{n(n+1) / 2}} \prod_{j=0}^{n-1} \frac{\Gamma\left(k-\frac{n-1}{2}+\frac{j}{2}\right)}{\Gamma\left(k-n+\frac{j}{2}\right)} \\
& =\frac{1}{2^{n^{2}+2 n} \pi^{n(n+1) / 2}} \prod_{1 \leq i \leq j \leq n}(2 k-2 n+i+j-2) .
\end{aligned}
$$

Now, for each integer $r$ with $0 \leq r \leq n$, let $\Pi_{r}$ be the set of elements $u$ of $\Gamma_{n}(N)$ such that $u$ is $\operatorname{Sp}(n, \mathbb{Z})$-conjugate to a matrix of the form $\left(\begin{array}{cc}1_{n} & x \\ 0 & 1\end{array}\right)$, where $x \in L_{n}$ and is of rank $r$. When $r=0$, this is nothing but $1_{2 n}$. When $r \geq 1$, we call such unipotent elements central because they are $\operatorname{Sp}(n, \mathbb{Q})$ conjugate to some elements of the center of the unipotent radical of a maximal parabolic subgroup of $\operatorname{Sp}(n, \mathbb{Q})$. Using Shintani's notation, we denote by $I_{n}\left(\Pi_{r}, N, k\right)$ the contribution of $\Pi_{r}$ to $\operatorname{dim} S_{k}\left(\Gamma_{n}(N)\right)$. That is, we put

$$
I_{n}\left(\Pi_{r}, N, k\right)=\frac{a_{n}(k)}{\# Z\left(\Gamma_{n}\right)} \int_{\Gamma_{n} \backslash H_{n}} \sum_{\gamma \in \Pi_{r}} H_{\gamma}(Z) d Z .
$$

Shintani [21] has given a formula to express each $I_{n}\left(\Pi_{r}, N, k\right)$ as a product of some elementary factors and a special value of $\zeta\left(s, L_{n}^{*}, 1,1\right)$ as follows. For each positive integer $l$, we put

$$
\omega=\prod_{i=1}^{l} \zeta(2 i)
$$

and

$$
U_{l}=\prod_{i=1}^{l} \frac{2 \pi^{i}}{\Gamma(i)}
$$

THEOREM 8 (Shintani [21]). For each positive integer $n$ and each integer $k>2 n$, we have

$$
I_{n}\left(\Pi_{0}, N, k\right)=\left[\Gamma_{n}(1): \Gamma_{n}(N)\right] \frac{\omega_{n}}{U_{n}(4 \pi)^{n(n+1) / 2}} \prod_{i=1}^{n} \prod_{j=0}^{i-1}(2 k-n-i+2 j),
$$


and for each $r$ with $1 \leq r \leq n-1$, we have

$$
\begin{aligned}
I_{n}\left(\Pi_{r}, N, k\right)= & {\left[\Gamma_{n}(1): \Gamma_{n}(N)\right] \frac{\omega_{n-r}}{U_{n}(4 \pi)^{(n-r)(n-r+1) / 2}} } \\
& \times \prod_{i=1}^{n-r} \prod_{j=0}^{i-1}(2 k-n-i+2 j) \zeta\left(r-n, L_{r}^{*}, 1,1\right) .
\end{aligned}
$$

REMARK. In Shintani's original theorem, he assumed that $k \geq 2 n+3$, but it is easy to show that this also holds for $k \geq 2 n+1$ by applying carefully the estimation he used. Incidentally, $\zeta\left(s, L_{n}^{*}, 1,1\right)=\zeta_{r}^{*}(s)$ in his notation.

Using Theorem 8, we obtain an explicit formula for $I_{n}\left(\Pi_{r}, N, k\right)$ as a corollary to our Theorems 5 and 6 . We state this below.

Before stating this result, we prepare more notation. For each nonnegative integer $r$ with $0 \leq r \leq n$, we set

$$
\begin{aligned}
C_{n-r} & =C_{n-r}(k, N) \\
& =\left[\operatorname{Sp}(n, \mathbb{Z}): \Gamma_{n}(N)\right] N^{-r(n-(r-1) / 2)} \prod_{t=1}^{n-r} \frac{t !}{(2 t-1) ! !}\left(\begin{array}{c}
k-1-\frac{n}{2}+\frac{t}{2} \\
t
\end{array}\right),
\end{aligned}
$$

where, for any positive integer $t$, we denote by $\left(\begin{array}{c}\alpha \\ t\end{array}\right)$ the binomial coefficient

$$
\left(\begin{array}{l}
\alpha \\
t
\end{array}\right)=\frac{\alpha(\alpha-1) \cdots(\alpha-t+1)}{t !},
$$

and $(2 t-1) ! !=1 \cdot 3 \cdots(2 t-1)=2^{-t} t !\left(\begin{array}{c}2 t \\ t\end{array}\right)$. When $r=n$, the product part in the above definition of $C_{n-r}$ is regarded as 1 .

THEOREM 9. For any positive integers $n, N, k$ with $k>2 n$, the contribution $I_{n}\left(\Pi_{r}, k, N\right)$ is given as follows.

(1) For odd $r$ with $1 \leq r \leq n$,

$$
\begin{aligned}
& I_{n}\left(\Pi_{r}, k, N\right) \\
& =C_{n-r} \\
& \quad \times \frac{(-1)^{\left[\frac{n+1}{2}\right]}\left|B_{n-\frac{r-1}{2}} B_{2} B_{4} \cdots B_{r-1} B_{2} B_{4} \cdots B_{2 n-2 r} B_{2 n-2 r+2} B_{2 n-2 r+4} \cdots B_{2 n-r-1}\right|}{2^{n+\frac{r-1}{2}}\left(n-\frac{r-1}{2}\right) !\left(\frac{r-1}{2}\right) !} .
\end{aligned}
$$

In particular, if $n-(r-1) / 2$ is an odd number greater than 1 , then $I_{n}\left(\Pi_{r}\right.$, $k, N)=0$. 
(2) For even $r$ with $0 \leq r \leq n$,

$$
\begin{aligned}
& I_{n}\left(\Pi_{r}, k, N\right) \\
& =C_{n-r} \\
& \quad \times \frac{(-1)^{r\left(1+\delta_{n r}\right) / 2}\left|B_{2} B_{4} \cdots B_{r-2} B_{\frac{r}{2}}\right| \cdot\left|B_{2} B_{4} \cdots B_{2 n-2 r} B_{2 n-2 r+2} B_{2 n-2 r+4} \cdots B_{2 n-r}\right|}{2^{2 n-r / 2}\left(\frac{r}{2}\right) !\left(n-\frac{r}{2}\right) !},
\end{aligned}
$$

where $\delta_{n r}$ is the Kronecker symbol. In particular, for $r$ such that $r \equiv 2 \bmod 4$ with $r \neq 2$, we have $I_{n}\left(\Pi_{r}, k, N\right)=0$.

The proof is straightforward if we note that $\pi^{-i} \Gamma(i) \zeta(2 i)=\left(\left(2^{i-1}\right) /\right.$ $((2 i-1) ! !)) \pi^{i}\left|B_{2 i}\right|$.

When $N \geq 3$, it is conjectured, independently by several mathematicians, that any contribution to $\operatorname{dim} S_{k}\left(\Gamma_{n}(N)\right)$ of conjugacy classes other than central unipotent conjugacy classes and the identity element should vanish. So, we are naturally led to the following conjecture. The point of our conjecture is that everything is explicit.

Conjecture. When $N \geq 3$ and $k>2 n$,

$$
\operatorname{dim} S_{k}\left(\Gamma_{n}(N)\right)=\sum_{r=0}^{n} I_{n}\left(\Pi_{r}, k, N\right),
$$

where each $I_{n}\left(\Pi_{r}, k, N\right)$ is as given explicitly in Theorem 5 above.

This conjecture is known to be true for $n \leq 3$ by Christian [1] and [2], Morita [11], Yamazaki [25], and Tsushima [24].

\section{Numerical examples of the conjecture}

We assume that $N \geq 3$ hereafter.

(1) When $n=4$ and $k>8$, the conjecture reads

$$
\begin{aligned}
\operatorname{dim} & S_{k}\left(\Gamma_{4}(N)\right) \\
= & \left.\operatorname{Sp}(4, \mathbb{Z}): \Gamma_{4}(N)\right] \\
& \times\left(\frac{1}{2^{25} \cdot 3^{8} \cdot 5^{4} \cdot 7^{2}}(2 k-2)(2 k-3)(2 k-4)^{2}\right. \\
& \times(2 k-5)^{2}(2 k-6)^{2}(2 k-7)(2 k-8) \\
& +\frac{1}{N^{4}} \times \frac{1}{2^{17} \cdot 3^{7} \cdot 5^{3} \cdot 7}(2 k-3)(2 k-4)(2 k-5)^{2}(2 k-6)(2 k-7) \\
& \left.-\frac{1}{N^{7}} \times \frac{1}{2^{15} \cdot 3^{5} \cdot 5 \cdot 7}(2 k-4)(2 k-5)(2 k-6)+\frac{1}{N^{10}} \times \frac{1}{2^{12} \cdot 3^{4} \cdot 5}\right) .
\end{aligned}
$$


(2) When $n=5$ and $k>10$, the conjecture reads

$$
\begin{aligned}
\operatorname{dim} & S_{k}\left(\Gamma_{5}(N)\right) \\
= & {\left[\operatorname{Sp}(5, \mathbb{Z}): \Gamma_{5}(N)\right] } \\
& \times\left(\frac{(2 k-2)(2 k-3)(2 k-4)^{2}(2 k-5)^{2}(2 k-6)^{3}(2 k-7)^{2}(2 k-8)^{2}(2 k-9)(2 k-10)}{2^{33} \cdot 3^{12} \cdot 5^{5} \cdot 7^{3} \cdot 11}\right. \\
& -\frac{1}{N^{9}} \times \frac{1}{2^{23} \cdot 3^{7} \cdot 5^{3} \cdot 7}(2 k-4)(2 k-5)(2 k-6)^{2}(2 k-7)(2 k-8) \\
& -\frac{1}{N^{12}} \times \frac{1}{2^{17} \cdot 3^{7} \cdot 5^{2} \cdot 7}(2 k-5)(2 k-6)(2 k-7) \\
& \left.+\frac{1}{N^{14}} \times \frac{1}{2^{16} \cdot 3^{6} \cdot 5 \cdot 7}(2 k-6)\right) .
\end{aligned}
$$

Acknowledgments. We would like to thank the referee for the very careful reading and suggestions. In particular, Section 3.3 was added to answer a problem raised by the referee.

\section{REFERENCES}

[1] U. Christian, Berechnung des Ranges der Schar der Spitzenformen zur Modulgruppe zweiten Grades und Stufe $q>2$, J. Reine Angew. Math. 277 (1975), 130-154.

[2] - Zur Berechnung des Ranges der Schar der Spitzenformen zur Modulgruppe zweiten Grades und Stufe $q>2$, J. Reine Angew. Math. 296 (1977), 108-118.

[3] H. Cohen, Sums involving the values at negative integers of L-functions of quadratic characters, Math. Ann. 217 (1975), 271-285.

[4] D. Goldfeld and J. Hoffstein, Eisenstein series of $1 / 2$-integral weight and the mean value of real Dirichlet L-series, Invent. Math. 80 (1985), 185-208.

[5] T. Ibukiyama and H. Katsurada, "Koecher-Maass series for real analytic Siegel Eisenstein series" in Automorphic Forms and Zeta Functions (Tokyo, 2004), World Sci., Hackensack, NJ, 2006, 170-197.

[6] T. Ibukiyama and H. Saito, On zeta functions associated to symmetric matrices and an explicit conjecture on dimension of Siegel modular forms of general degree, Int. Math. Res. Not. IMRN 1992, no. 8, 161-169.

[7] - On zeta functions associated to symmetric matrices, I: An explicit form of zeta functions, Amer. J. Math. 117 (1995), 1097-1155.

[8] - On zeta functions associated to symmetric matrices, III: An explicit form of L-functions, Nagoya Math. J. 146 (1997), 149-183.

[9] — On "easy" zeta functions [translation of Sūgaku 50 (1998), 1-11], Sugaku Expositions 14 (2001), 191-204.

[10] W. Kohnen, Modular forms of half-integral weight on $\Gamma_{0}(4)$, Math. Ann. 248 (1980), 249-266.

[11] Y. Morita, An explicit formula for the dimension of spaces of Siegel modular forms of degree two, J. Fac. Sci. Univ. Tokyo Sect. IA Math. 21 (1974), 167-248.

[12] H. Saito, Explicit form of the zeta functions of prehomogeneous vector spaces, Math. Ann. 315 (1999), 587-615. 
[13] I. Satake, "On zeta functions associated with self-dual homogeneous cones" in Number Theory and Related Topics (Bombay, 1988), Tata Inst. Fund. Res. Stud. Math. 12, Tata Inst. Fund. Res., Bombay, 1989, 177-193.

[14] I. Satake and J. Faraut, The functional equation of zeta distributions associated with formally real Jordan algebras, Tohoku Math. J. (2) 36 (1984), 469-482.

[15] I. Satake and S. Ogata, "Zeta functions associated to cones and their special values" in Automorphic Forms and Geometry of Arithmetic Varieties, Adv. Stud. Pure Math. 15, Academic Press, Boston, 1989, 1-27.

[16] F. Satō, On zeta functions of ternary zero forms, J. Fac. Sci. Univ. Tokyo Sect. IA Math. 28 (1981), 585-604.

[17] M. Sato and T. Shintani, On zeta functions associated with prehomogeneous vector spaces, Ann. of Math. (2) 100 (1974), 131-170.

[18] G. Shimura, "Modular forms of half integral weight" in Modular Forms of One Variable, I (Antwerp, 1972), Lecture Notes in Math. 320, Springer, Berlin, 1973, 57-74.

[19] - On modular forms of half integral weight, Ann. of Math. (2) 97 (1973), 440-481.

[20] - On the holomorphy of certain Dirichlet series, Proc. Lond. Math. Soc. (3) 31 (1975), 79-98.

[21] T. Shintani, On zeta functions associated with the vector space of quadratic forms, J. Fac. Sci. Univ. Tokyo Sect. IA Math. 22 (1975), 25-65.

[22] C. L. Siegel, Über die Zetafunktionen indefiniter quadratischer Formen, Math. Z. 43 (1938), 682-708.

[23] J. Sturm, Special values of zeta functions, and Eisenstein series of half integral weight, Amer. J. Math. 102 (1980), 219-240.

[24] R. Tsushima, A formula for the dimension of spaces of Siegel cusp forms of degree three, Amer. J. Math. 102 (1980), 937-977.

[25] T. Yamazaki, On Siegel modular forms of degree two, Amer. J. Math. 98 (1976), $39-53$.

[26] A. Yukie, Shintani Zeta Functions, London Math. Soc. Lecture Note Ser. 183, Cambridge University Press, Cambridge, 1993.

[27] D. B. Zagier, "Modular forms whose coefficents involve zeta functions of quadratic fields" in Modular Forms of One Variable, VI (Bonn, 1976), Lecture Notes in Math. 627, Springer, Berlin, 1977, 105-169.

Tomoyoshi Ibukiyama

Department of Mathematics

Graduate School of Science

Osaka University

Osaka 560-0043

Japan

ibukiyam@math.sci.osaka-u.ac.jp

Hiroshi Saito

(deceased) 